\title{
1 Genomic Rearrangements Considered as Quantitative Traits
}

2

3 Martha Imprialou' ${ }^{1}$, André Kahles ${ }^{2}$, Joshua G. Steffen ${ }^{3}$, Edward J. Osborne $^{3}$,

4 Xiangchao Gan ${ }^{4}$, Janne Lempe ${ }^{4}$, Amarjit Bhomra $^{1}$, Eric Belfield ${ }^{5}$, Anne

$5 \quad$ Visscher $^{5,6}$, Robert Greenhalgh ${ }^{3}$, Nicholas P Harberd $^{5}$, Richard Goram ${ }^{7}$,

6 Jotun Hein ${ }^{8}$, Alexandre Robert-Seilaniantz ${ }^{9}$, Jonathan Jones ${ }^{10}$, Oliver

$7 \quad$ Stegle $^{11}$, Paula Kover ${ }^{12}$, Miltos Tsiantis ${ }^{4}$, Magnus Nordborg ${ }^{13}$, Gunnar

8 Rätsch ${ }^{2}$, Richard M. Clark ${ }^{3,14}$, Richard Mott ${ }^{1,15}$

9

$10{ }^{1}$ Wellcome Trust Centre for Human Genetics, University of Oxford, OX3 7BN,

11 UK

$12{ }^{2}$ Memorial Sloan-Kettering Cancer Center, New York City, NY 10065, USA

$13{ }^{3}$ Department of Biology, University of Utah, Salt Lake City, UT, 84112-0840,

14 USA

$15{ }^{4}$ Max Planck Institute for Plant Breeding Research, 50829 Köln, Germany

$16{ }^{5}$ Department of Plant Sciences, University of Oxford, Oxford, OX1 3RB, UK

$17{ }^{6}$ Department of Comparative Plant and Fungal Biology, Royal Botanic

18 Gardens Kew, Ardingly, RH17 6TN, UK

$19{ }^{7}$ John Innes Centre, Norwich, NR4 7UH, UK

$20{ }^{8}$ Department of Statistics, University of Oxford, OX1 3TG, Oxford, UK

$21{ }^{9}$ UMR INRA-Agrocampus Ouest-Université de Rennes 1, 35653 Le Rheu

22 Cedex, France

$23{ }^{10}$ The Sainsbury Laboratory, Norwich Research Park, Norwich NR4 7UH, UK.

$24{ }^{11}$ European Bioinformatics Institute, Hinxton, Cambridge, CB10 1SD, UK 
$1{ }^{12}$ Dept of Biology and Biochemistry, University of Bath, Bath, BA2 7AY, UK

$2{ }^{13}$ Gregor Mendel Institute of Molecular Plant Biology, Vienna, 1030, Austria

$3{ }^{14}$ Center for Cell and Genome Science, University of Utah, Salt Lake City, UT,

4 84112-0840, USA

$5 \quad{ }^{15}$ UCL Genetics Institute, University College London, WC1 6BT, UK

6 


\section{Abstract}

3 To understand the population genetics of structural variants (SVs), and their

4 effects on phenotypes, we developed an approach to mapping SVs,

5 particularly transpositions, segregating in a sequenced population, and which

6 avoids calling SVs directly. The evidence for a potential SV at a locus is

7 indicated by variation in the counts of short-reads that map anomalously to the

8 locus. These SV traits are treated as quantitative traits and mapped

9 genetically, analogously to a gene expression study. Association between an

10 SV trait at one locus and genotypes at a distant locus indicate the origin and

11 target of a transposition. Using ultra-low-coverage (0.3x) population

12 sequence data from 488 recombinant inbred Arabidopsis genomes, we

13 identified 6,502 segregating SVs. Remarkably, $25 \%$ of these were

14 transpositions. Whilst many SVs cannot be delineated precisely, PCR

15 validated $83 \%$ of 44 predicted transposition breakpoints. We show that

16 specific SVs may be causative for quantitative trait loci for germination, fungal

17 disease resistance and other phenotypes. Further we show that the

18 phenotypic heritability attributable to sequence anomalies differs from, and in

19 the case of time to germination and bolting, exceeds that due to standard

20 genetic variation. Gene expression within SVs is also more likely to be

21 silenced or dysregulated. This approach is generally applicable to large

22 populations sequenced at low-coverage, and complements the prevalent

23 strategy of SV discovery in fewer individuals sequenced at high coverage. 


\section{Introduction}

3 Whilst genome resequencing has become cheap and ubiquitous, and can

4 readily determine variations such as SNPs and very small indels, the problem

5 of identifying structural variants (SVs) and rearrangements remains a

6 challenge, despite continual improvement in algorithms for calling SVs. The

7 current gold standard for determining SVs between individuals is by de-novo

8 assembly ${ }^{1}$. This requires very high-coverage paired-end sequence over a

9 range of insert sizes, together with long-range information for scaffolding.

10 Advances in long-read technologies ${ }^{2,3}$ are beginning to aid this process, but

11 the relatively high cost and low throughput of this strategy limits its

12 applicability to smaller numbers of genomes, and leaves open two important

13 questions. First, whether an SV identified in an individual is unique, or is

14 frequent enough to contribute appreciably to phenotypic heritability in a

15 population. Second, whether an SV represents a local rearrangement, such

16 as a deletion, inversion or tandem copy-number variant (CNV), or is long-

17 range, such as a transposition ${ }^{4,5}$.

19 SVs are often revealed by the anomalous alignment of short-reads to the

20 reference genome. Specific anomaly signatures characterize different types of

21 SVs (Table 1). Thus, same-strand pairs indicate inversion, high read coverage

22 duplications, abnormal insert sizes and unpaired reads are classified as

23 indels. 
1 These signatures include excess read coverage (e.g., duplications, Copy

2 Number Variants $(\mathrm{CNV}))$, discordant distances between read pairs (e.g.,

3 indels) and inconsistent read orientation (e.g. inversions). These anomalies

4 arise, often in combination, because the reads have been aligned to the

5 wrong genome - the anomalies should disappear if instead the reads were

6 aligned to the true genome. This idea is used by algorithms such as GATK ${ }^{6}$

7 and Platypus ${ }^{7}$ that identify small indels by local realignment, and in whole-

8 genome reassembly by iterative realignment ${ }^{8}$.

9

10 Many SV-calling algorithms utilize these read-anomaly signatures to identify

11 SVs segregating in individuals sequenced at high coverage ${ }^{9-16}$. These

12 methods focus on short-range SVs because of the difficulties in distinguishing

13 long-range rearrangements from read mapping errors. They are also designed

14 to work best when calling SVs in individuals sequenced at intermediate to high

15 coverage; for example, two of the most recent SV-callers, LUMPY ${ }^{15}$ and

$16 \mathrm{WHAM}^{16}$ are most sensitive when sequence coverage is at least $10 \mathrm{x}$. In other

17 applications, e.g cancer resequencing, typical coverage is even higher, at $30 x$

18 or above.

20 The problem of calling SVs from population sequence data presents additional

21 challenges. Population studies are generally conducted for the purpose of

22 genetic association, and consequently require large sample sizes. Population

23 sequencing provides an alternative to genotyping by SNP arrays,

24 simultaneously providing both haplotype reference panels for imputation ${ }^{17}$ and 
1 cohorts for disease mapping ${ }^{18,19}$. As the sample size increases, it becomes

2 possible to reduce the coverage of each individual dramatically, yet still

3 impute single nucleotide polymorphism (SNPs) accurately ${ }^{20}$. Consequently

4 one would want to be able to call SVs as well as SNPs and to test them for

5 association. Although the information present in each sample is sparse, and

6 therefore it would be difficult to call SVs (and SNPs) on an individual basis, by

7 pooling information across samples it might be possible to determine common

8 SVs analogously to the way SNPs are imputed.

9

10 A further challenge, which is not confined to low-coverage sequencing, is that

11 presented by complex SVs. Unlike simple indels, inversions and

12 transpositions, where a segment with well-defined breakpoints is affected,

13 many SVs are composites of multiple events ${ }^{21}$, often driven by transposons

14 and other repetitive mobile elements. Complex SVs resist simple

15 classification, and it may be impossible to determine the precise sequence of

16 mutations that occurred in the lineages separating the reference genome from

17 that of the sequenced individual. Whilst current algorithms for calling SVs in

18 simulated high-coverage human data can identify simple SVs with sensitivities

19 of about $90 \%$ depending on the type of SV ${ }^{16}$, they are less accurate when

20 applied to real data, and their performance on complex SVs is unreported.

22 Nonetheless, even though it may be difficult to delineate complex SVs, there

23 can still be strong evidence from read-mapping anomalies that an SV of some

24 sort exists at a locus. If the intensity of its anomaly signature can be used as a 
1 proxy for the purpose of testing genetic association, then one need not

2 delineate the SV precisely. It then follows that the genomewide information

3 captured by these anomalies could be used to compute relationships between

4 individuals based on their structural similarities alone, and hence to estimate

5 the heritability attributable to this source of variation.

6

7 Here, we ask whether low-coverage population sequencing provides new

8 ways for mapping SVs and estimating heritability, complementing the

9 sequencing of fewer individuals at high coverage. As an illustration, we

10 investigate the architecture and phenotypic impact of structural variation in

11 Arabidopsis thaliana. Among natural accessions of Arabidopsis, structural

12 variation is plentiful ${ }^{22}$. The extent of rDNA repeats ${ }^{23}$ and mobile transposable

13 elements ${ }^{24}$ vary between accessions, and variation in the overall amounts of

14 both classes of repetitive sequence elements are complex traits, partially

15 under genetic control. In this study we investigate all types of structural

16 variation in Arabidopsis, including those not mediated by mobile elements.

17 We show that long-range transpositions are common, and that structural

18 variation has a significant impact on particular quantitative trait loci (QTLs)

19 and on trait heritability, distinct from that explained by other types of sequence

20 variation.

\section{Results}




\section{Structural Variants as Quantitative Traits}

3 We combined established ideas from signature-based SV identification with

4 quantitative genetics to analyse structural variation in population sequence

5 data. The following scenario motivates our reasoning: suppose an SV arose in

6 a certain population ancestor, $\alpha$, transposing a genomic segment $s$ originating

7 at a "source" locus $L$ and targeting to a "sink" locus $M$. Source and sink can be

8 coincident or unlinked, but for the moment, suppose they are unlinked. If the

9 event is transposon-mediated, then the segment $s$ is duplicated to $s^{\prime}$ at $M$, and

10 possibly altered, leaving the original $s$ at $L$. Once random chromosomal

11 assortment and recombination has occurred, in the present-day population

12 there will be a mix of individuals carrying the segment at neither, one or both

13 loci.

15 In the descendent population, one individual is sequenced and becomes the

16 reference genome. Depending on the choice of reference individual and the

17 mechanism of transposition, the reference might carry zero or one copies of $s$ 18 at the source and of $s^{\prime}$ at the sink.

20 Assume the reference has one copy of $s$ and zero copies of $s^{\prime}$. A population

21 sample will contain individuals with mix of all possible configurations at source 22 and sink. But only individuals that inherited the haplotype descended from $\alpha$ 23 at the sink carry the transposed segment, regardless of their haplotype at the 24 source. The individuals are sequenced with short-reads, and the reads are 
1 mapped to the reference genome. Individuals carrying the transposition s' at

2 the sink will have reads spanning the breakpoint that split between source and

3 sink. Hence read mapping anomalies apparently originating at the source will

4 be enriched in those individuals carrying the sink haplotype $\alpha$ : genotypes that

$5 \quad \operatorname{tag} \alpha$ at the sink will be associated with anomalies at the source.

6

7 If on the other hand the reference contains both $s$ at the source and $s^{\prime}$ at the

8 sink then those individuals that did not inherit the haplotype $\alpha$ at the sink will

9 appear to carry a deletion there. Reads with anomalously large insert sizes

10 will map to the sink and will be associated with genotypes tagging the

11 haplotype $\alpha$ at the sink - the generative role played by the source will be 12 invisible.

14 Similarly, by considering situations where the source and sink are coincident -

15 for example tandem duplications - in a population we would expect to 16 encounter a mix of short-range cis and long-range trans associations between 17 different classes of read-mapping anomalies and genotypes, depending on 18 the diverse histories of each structural variants. 


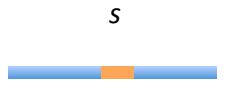

source $L$

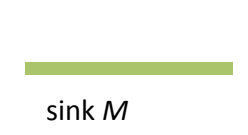

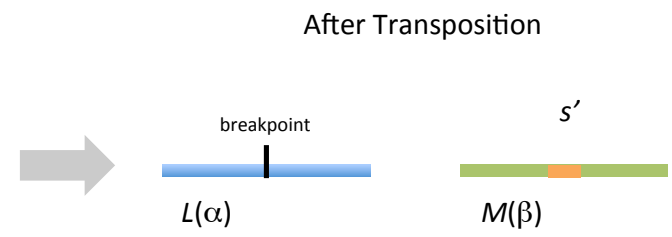

\section{Present-Day Population Sample}

Reality

a

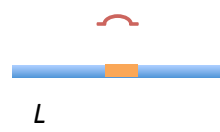

b

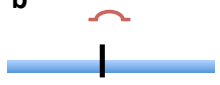

$L(\alpha)$

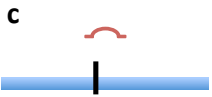

$L(\alpha)$

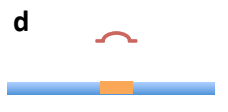

L
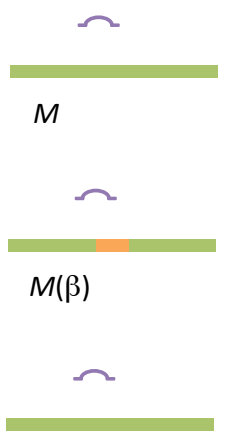

$M$

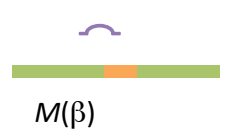

Appearance after alignment to reference

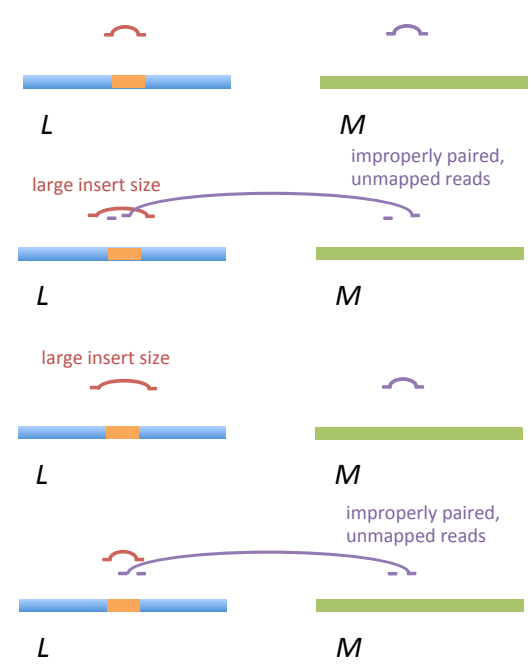

1

Figure 1 Effects of a transposition on short-read mapping. Chromosomes are horizontal bars and read pairs are pairs of horizontal lines linked by curves. Upper shows a population ancestor corresponding to the reference genome (left) undergoing a transposition (right), in which a segment $s$ at source locus $L$ with haplotype context $\alpha$ is copied to $s^{\prime}$ at recipient sink locus $M$ with haplotype context $\beta$. Lower shows all four possible combinations (a-d) of source $L$ and sink $M$ haplotype in descendants. On left are shown the alignment of reads to the true haplotypes, where there are no read-mapping anomalies. On right are shown the various read-mapping anomalies that arise, depending on the true haplotype backgrounds at source and sink, upon alignment to the reference genome. 
1 To apply these ideas in practice, we count the numbers of anomalous reads

2 mapping to each source $L$ in a population sample, treat it as a quantitative

3 trait, and proceed to identify genetic loci containing variation that correlate

4 with variation in the trait. This procedure defines a SV quantitative trait locus

5 (SV-QTL) linking $T_{L i}$, the number of anomalous reads mapping to locus $L$ in

6 individual $i$ and the haplotype $H_{M i}$ at $\operatorname{sink}$ locus $M$ in individual $i$ (Figure 1,

7 Methods). cis SV-QTLs where the source and sink overlap indicate local

8 structural variants such as CNVs, deletions and inversions; trans SV-QTLs

9 indicate transpositions (insertional translocations) or larger scale

10 rearrangements. In this way we can determine whether an SV is in trans, its

11 originating haplotype, which individuals now carry it (Figure S1), and its

12 frequency (Figure S2).

14 We interpret the matrix of SV-traits across all loci as a Euclidean 15 representation of haplotype space, in the sense that, if two individuals are 16 genetically similar then their SV-trait vectors should be close together. 17 Consequently we define a genome-wide similarity between individuals based 18 on the similarity of their anomalies, as a weighted average of their locus19 specific similarities. Taken across all individuals, these generate a structural 20 variation similarity matrix, analogous to a SNP-based genetic relationship 21 matrix. This matrix was used to estimate the heritability of a phenotype with 22 respect to structural variation, and compared to the heritability associated with 23 SNP variation. 


\section{Structural Variation in Arabidopsis}

2

3 We used our strategy to map cis and trans SVs in the $120 \mathrm{Mb}$ genome of the

4 plant Arabidopsis thaliana. We sequenced 488 of the Arabidopsis Multiparent

5 Advanced Generation Inter-Cross (MAGIC) recombinant inbred lines ${ }^{25}$ at $6 \sim 0.3 \mathrm{x}$ coverage using $51 \mathrm{bp}$ paired-end Illumina reads. The MAGIC lines

7 descend from 19 ancestral founder accessions that have been sequenced at 8 high coverage ${ }^{8}$ (Table S1) such that each $\sim 120 \mathrm{Mb}$ genome is a mosaic of the

919 founder haplotypes. Consequently we expect most SVs segregating in

10 MAGIC to also segregate in the founders, thereby providing a means to verify

11 any SVs we detect. The choice of MAGIC lines rather than natural accessions

12 means that the confounding effects of population structure and of selection

13 are largely absent from the population. Very rare alleles with frequency below

$141 / 19=4.5 \%$ are uncommon, increasing the power to detect QTLs. However, 15 MAGIC QTL mapping resolution is also poorer, at $\sim 200 \mathrm{~kb}$, compared to $\sim 10 \mathrm{~kb}$ 16 in natural accessions.

18 We mapped the reads to the TAIR10 reference using Stampy ${ }^{26,27}$ and 19 inferred the mosaic of each line using a hidden Markov model (HMM) 20 implemented in the software 'reconstruction' available on request from the 21 authors. The algorithm uses as input SNP calls for each MAGIC genome, and 22 the set of of $1.2 \mathrm{M}$ biallelic variants in the 19 founders (excluding loci tagged as 23 within transposons, and those sites called as heterozygous or multi-allelic in 24 the founders $)^{17}$, and finds the most likely sequence of haplotype assignments 
1 for each chromosome. Because the lines were called at low coverage, most

2 SNP sites were not covered by reads in an given; consequently we called on

3 average $301 \mathrm{k}$ SNPs per line (using GATK $^{6}$ ) (ie a randomly sampled of $\sim 25 \%$

4 of the 1.2M sites). However, this amount of data is sufficient for the HMM to

5 determine the founder mosaic accurately; we estimated by simulation that the

6 algorithm can delineate the mosaic breakpoints (which correspond to

7 recombination events) to within $\sim 2 \mathrm{~kb}$ (data not shown).

8

9 Using this procedure, we reconstructed each MAGIC genome into $~ 34$

10 haplotype blocks on average with mean size $3.48 \mathrm{Mb}$, representing

11 contributions from about 11 founder haplotypes (Table S2), and imputed the

12 full variant catalogue into each lines. Comparison of imputed SNPs with 782

13 GoldenGate SNP genotypes measured in 370 of the MAGIC lines ${ }^{25}$ showed $1498 \%$ concordance.

16 To map SVs, we divided the reference genome into 11,915 abutting source 17 loci, each $10 \mathrm{~kb}$ wide, and computed six measures of anomalous read 18 mapping in each locus $\left(6^{\star} 11,915=71,490\right.$ SV trait vectors) (Methods, Table

19 1a, Table S3). Four of these measures address different types of anomalous 20 read mapping that provide evidence of specific anomalies, namely high read 21 coverage for duplications, strandedness of reads for inversions, anomalously 22 large insert size for translocations and unpaired reads for deletions. The 23 remaining two measures are linear combinations of other measures that could 24 co-exist. 
(a)

\begin{tabular}{lrrrr}
\hline trait type & SV-QTLs & Unique & cis & trans \\
\hline IP & 1997 & 833 & 1617 & 380 \\
ER & 184 & 165 & 112 & 72 \\
LIS & 2051 & 585 & 1677 & 374 \\
SS & 1950 & 1887 & 1358 & 592 \\
U & 2060 & 1998 & 1530 & 530 \\
U+LIS & 2033 & 431 & 1661 & 372 \\
\hline Total & 10275 & 5899 & 7955 & 2320
\end{tabular}

(b)

\begin{tabular}{lrrr}
\hline SV type & SV-QTLS & cis & trans \\
\hline duplication & 175 & 109 & 66 \\
indel & 3035 & 3035 & 0 \\
inversion & 1976 & 1373 & 603 \\
other & 1316 & 381 & 935 \\
\hline Total & 6502 & 4898 & 1604
\end{tabular}

1 Table 1 (a) MAGIC SV-QTLs classified by read pair anomaly type. SV-QTLs:

2 total number of QTLs detected using each anomaly type (if the same QTL was

3 detected by multiple anomalies then it is counted multiple times in this

4 column), Unique: number of QTLs detected only by a single anomaly

5 category, cis: number of cis SV-QTLs, trans: number of trans SV-QTLs. (b)

6 MAGIC SV-QTLs classified by QTL type, after removing duplicates. SV-QTLs:

7 number of structural variants of each type, cis: number of cis SV-QTLs

8 (source and sink within $2 \mathrm{Mb}$ from each other), trans: number of trans SV-

9 QTLS [Note that the total number of SV-QTLS is 10,275 , of which 6,502 are

10 distinct after removing overlapping events, and 5,899 unique to a single

11 anomaly type.] 
1 Genetic association between each of the SV-trait vectors and the local

2 haplotype space was determined using a one-way ANOVA. We chose to

3 determine association at the level of haplotypes rather than SNPs for two

4 reasons. First, the founder haplotype space in the MAGIC lines is well-

5 defined, and measuring association with haplotypes can capture relationships

6 invisible at the level of SNPs. Second, the set of haplotype tests - defined by

7 the union of all the breakpoints, comprising 16,700 haplotype blocks, such

8 that the ancestral haplotype of all lines is unchanged within each block -

9 means about 75 times fewer tests are performed, thereby speeding up the

10 procedure (Methods). To determine genome-wide significance thresholds for

11 SV-QTLs we performed 100 phenotype permutations for each trait and then

12 fitted extreme value distributions (evd) to the genome-wide maxima of the

13 permutations (Methods). We merged together probable duplicate overlapping

14 SV-QTLs identified by multiple anomaly types.

16 After removing duplicates we identified 6,502 SV-QTLs at 1\% study-wide false

17 discovery rate (evd $\mathrm{P}<0.001$ ) (Table S3). Of these, 1,604 (25\%) were trans,

18 defined as mapping over $2 \mathrm{Mb}$ from the source. Overall, 4,073/11,915 (34.2\%)

19 source loci harboured structural variants. Whilst we have greater power to

20 detect larger SVs, 2,379 overlapped annotated indels shorter than $2 \mathrm{~kb}^{8}$. 

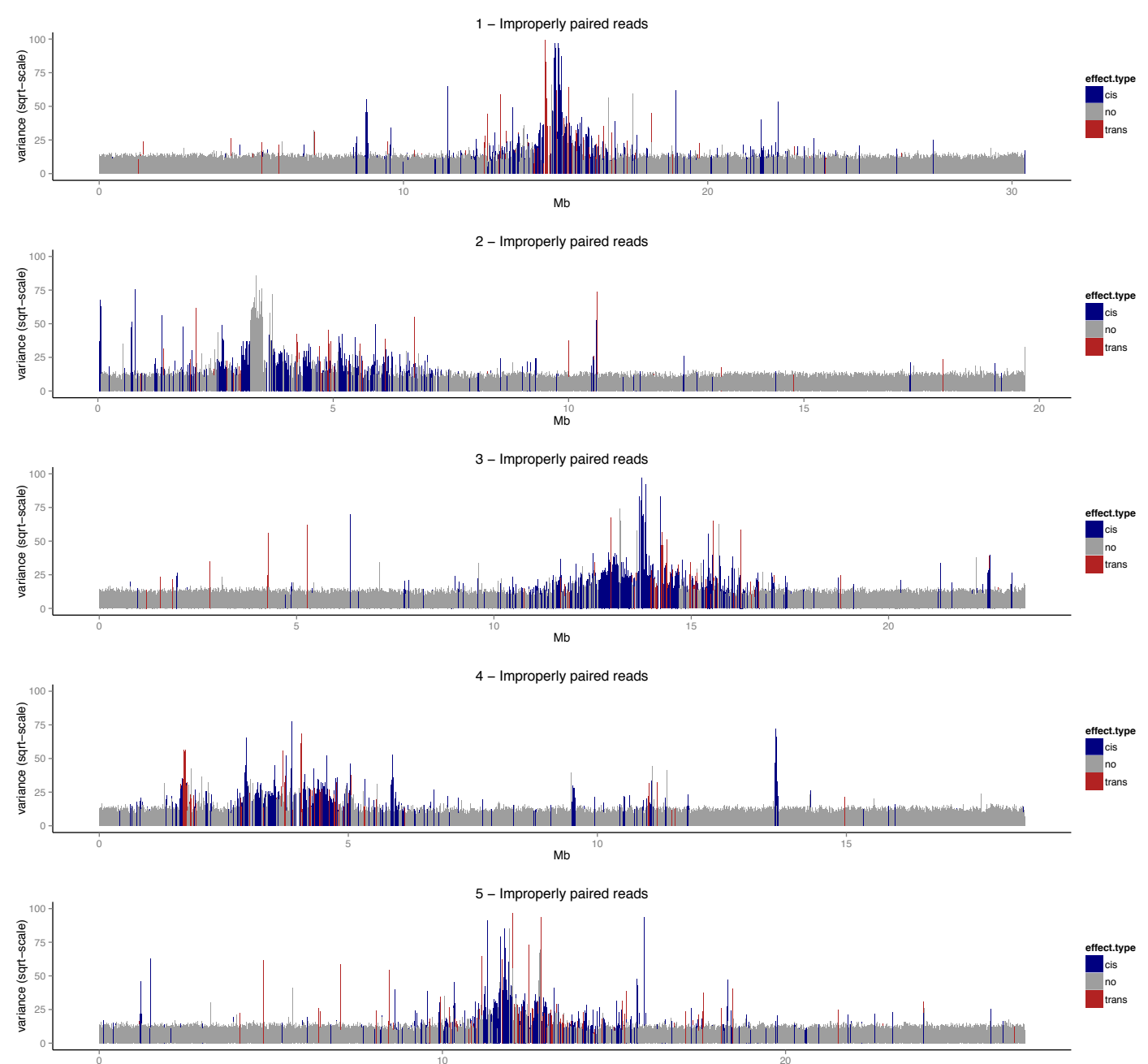

2 Figure 2 Genome-wide distribution of the variance for the trait "improperly3 paired reads" (the number of reads mapping to a locus with mapping 4 anomalies), computed in 10-kb windows. The $x$-axis shows genomic position 5 and the $y$-axis the variance of each trait vector scaled by its mean. Each 6 vertical line corresponds to a window. Those with SV-QTLs are blue (cis) and 7 red (trans). Centromeres are marked by pink bars.

9 The likelihood that a structural trait vector has an SV-QTL increases with its 10 variance (Figure 2). SV-QTLs are enriched around centromeres, as expected. 11 Away from the centromeres, Figure 2 also shows that bins with variable SV 12 traits are isolated, rather than in clusters. Figure 3a shows the genome-wide 
1 distribution of SVs segregating in one MAGIC founder, Ler-0. Figure 3 and

2 Table S3 show trans SV-QTLs link all five chromosomes.

3

4 In 319 SVs we were able to pinpoint both breakpoints, using contigs from de-

5 novo assemblies of the 19 founder genomes ${ }^{8}$ (see validation section below).

6 Mean SV size was $53 \mathrm{~kb}$ in these SVs, and the largest was $189 \mathrm{~kb}$. Thus the

7 many of the SVs we discovered are too large to be due to insertions of small

8 transposable elements. This probably reflects our lack of power to detect very

9 small events, but also emphasizes that not all SVs are driven by mobile 10 elements.

\section{Validation}

14 Genome-wide confirmation of SVs using short-read sequence is challenging 15 because SV breakpoints often associate with transposons and repeats that 16 hinder read-mapping and reassembly. However, among our SV-QTLs are 17 several known rearrangements. These include trans SV-QTLs linking a cluster 18 of rDNA repeats at $\sim 14.2 \mathrm{Mb}$ on chromosome three to clusters at the ends of 19 chromosome two. Polymorphisms in these clusters are implicated in massive 20 genome size variations among Arabidopsis accessions ${ }^{28}$. We also identified 21 the known knob inversion on chromosome 4 as reciprocal transpositions

22 linking $1.61 \mathrm{Mb}$ and $2.65 \mathrm{Mb}^{29}$, and a $93 \mathrm{~kb}$ inverted transposition identified 23 previously in a cross between Ler- 0 and $\mathrm{Col}-0^{30}$, and found it was present in 2412 MAGIC founders. 


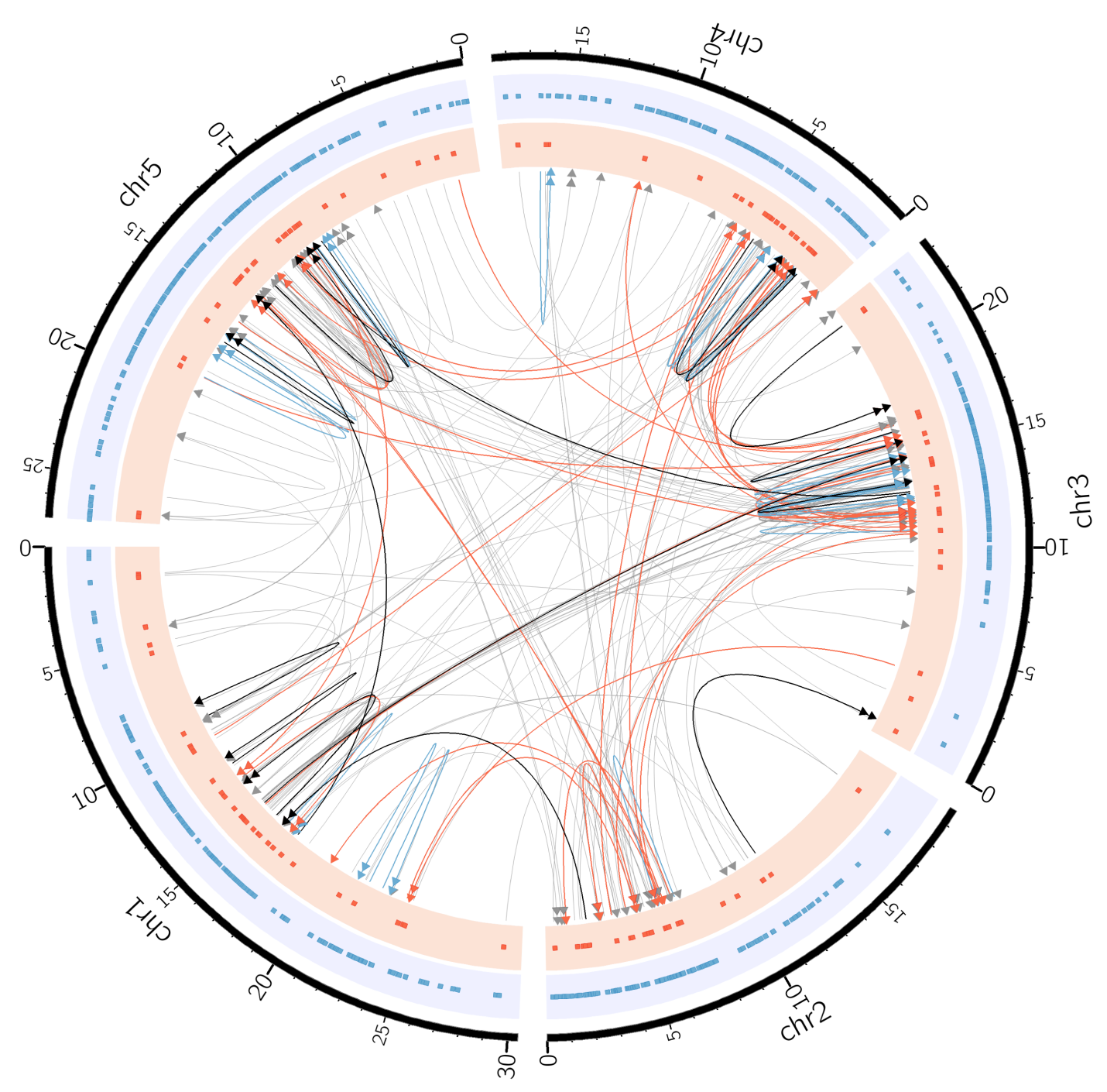

3 Figure 3 Structural variants segregating in the accession Ler-0. The grey 4 directed lines show SV-QTLs with the arrows pointing towards the sink locus. 5 Red and blue links indicate 37 trans and 30 cis SV-QTLs confirmed by de 6 novo contigs. The black links show 16 SVs confirmed by PCR ( 7 cis, 9 trans). 7 Double arrows in links indicate inversions. The dots in the red and blue tracks 8 mark the sources (trans and cis, respectively) of all SVs associated with the 9 Ler-0 haplotype.

10 To validate further SVs we compared our SV calls for the founder accession 11 Ler-0 against two Ler-0 contigs (chr3:16.65-17.02Mb, chr5:25.06-25.23Mb)

12 that were independently re-sequenced and manually reassembled ${ }^{31}$, thereby 13 constituting a gold standard for comparison. The chromosome 3 contig 
1 (Figure 4) is enriched in SVs (83 indels, 31 larger than 100bp), consistent

2 with our analysis: 42 SV-QTL sources (36 cis and 6 trans) are in this region

3 and 4 trans SV-QTLs map into it. As would be expected, the sources of these

4 SV-QTLs are within gaps in the contig. Furthermore, alignment revealed two

5 long-range SVs within the contig (a transposition and a duplication which align

6 to chromosomes four and two, respectively), which coincide with the source

7 and sink of two trans SV-QTLs mapped within the contig. Similarly, in the

8 chromosome 5 contig, 6 cis SV-QTLs correspond to deletions (Figure S3).

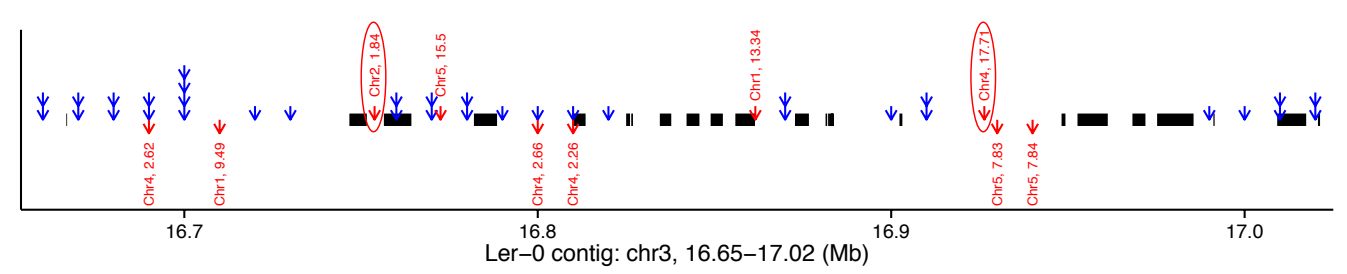

11 Figure 4 Alignment of a manually assembled contig from Ler-0, chr3:16.65-

$1217.02 \mathrm{Mb}$ to the reference annotated with SV-QTLs. Thick black lines show 13 alignments to reference genome. Blue arrows show the sources of cis SV14 QTLs; stacked arrows mean multiple read anomaly traits had SV-QTLs. Red 15 arrows display trans QTLs with arrows starting from the source and pointing 16 towards the sink. Gaps in the contig alignment indicate loci where Ler-0 did 17 not align to the reference, with the exception of two transposed segments that 18 mapped to chromosomes 2 and 4 at positions concordant with the sources of 19 two trans SV-QTLs (circled).

We also used an independent de-novo assembly of Ler-0 built from long PAC-

22 BIO reads, Genbank accession GCA_000835945.1 ${ }^{32}$ to validate our trans SV 23 predictions. This assembly was constructed algorithmically without manual 24 revisions, and so is not guaranteed to be correct. Further, the Ler-0 individual 
1 sequenced in the PAC-BIO assembly was different from the individual that

2 founded the MAGIC population and therefore might carry private structural

3 variations. Nonetheless, we expect it to be more accurate and contiguous

4 than a Ler-0 assembly built from short Illumina reads alone. We took those

53080 Illumina paired-end reads for Ler- 0 from ${ }^{8}$ that carried large insert size

6 mapping anomalies when mapped to TAIR10 and that mapped to the sources

7 of our predicted Ler-0 trans SV-QTLS, and then mapped them to the PAC-BIO

8 assembly using bwa $^{26}$. These Illumina reads are from an individual grown

9 from the same batch of seeds used to found the MAGIC population in 2007,

10 and should therefore share the same structural variants. Read anomalies that

11 gave rise to correct SV predictions should map contiguously to the PAC-BIO

12 assembly, under the assumption that the latter assembly is a more accurate

13 representation of the Ler-0 genome. We found $2460(80 \%)$ of these formerly

14 split Illumina read pairs now mapped contiguously, defined as both members

15 of a read-pair mapping to the PAC-BIO assembly with an insert size below $16600 \mathrm{bp}$.

18 With the exception of these manually assembled Ler-0 contigs and the

19 provisional Ler-0 PAC-BIO assembly, the MAGIC founders are not 20 contiguously reassembled into a genome-wide gold standard reference panel.

21 Nevertheless, they provide some information to test our SV predictions. To do 22 this, at each SV-QTL we predicted which founder haplotypes carried SVs at 23 the origination of the population. Using the low coverage data for the 488 24 MAGIC lines, at each SV-QTL we predicted which group of founders carried 
1 the SV allele vs the reference allele based on correspondence between their

2 SV-trait value and predicted founder allele, using the fact that SV haplotypes

3 will have elevated anomalous reads at the source. We were able to do this

4 confidently at 2,391 SVs where the founders divided into two groups, the

5 remainder having complex multi-allelic SV predictions (Methods). We then

6 examined the independently-collected high-coverage reads in each the 19

7 MAGIC founders ${ }^{8}$ for read-mapping signatures that supported the predicted

8 grouping of founders at each SV. We counted the read pairs linking source

9 and sink at each of the 2,391 SVs in the 19 high coverage founders. At

$101,585 / 2,391(66.3 \%$, FDR 7.5\%) SVs we observed significant differences in

11 anomalies between the predicted groupings of founders (Figure S4, which

12 also shows that the majority of SVs were mapped within $50 \mathrm{~kb})$. In the

13 founders, the mean SV allele frequency was $6 / 19=31 \%$. Only $387(12 \%)$ were

14 private to a single founder (Figure S2), in contrast to the fraction of SNPs

$15(45 \%)$ that are private to a single founder ${ }^{8}$.

17 A related analysis using low-coverage reads from the 488 MAGIC genomes, 18 but independent of founder predictions, (Methods) supported 1228/2391

19 (51.3\%, most also supported by the founder genomes) and 1631/4111

$20(39.7 \%)$ of those remaining SVs without founder predictions. In total $212,965 / 6,502(45.6 \%)$ SVs were supported by either method. 
1 In order to estimate SV sizes and identify SV breakpoints that could be tested

2 experimentally by PCR, we next assembled the high-coverage sequence data

3 for the MAGIC founders into de-novo contigs. No scaffolding was attempted in

4 order to produce conservative high-quality short contigs, each up to a few

5 kilobases long. We aligned these contigs to the reference to find alignments

6 split between sources and sinks. We mapped breakpoints for 420 SV-QTLs

7 (Methods, Table S4): in 319 SV-QTLs both breakpoints were identified. We

8 designed PCR primers around 77 breakpoints from 45 SVs (both breakpoints

9 in 7 SVs, and one in each of the remaining 38). We validated 37 SVs (83\%),

10 comprising 61 (79\%) breakpoints, in 14 cis (6 inversions, 7 transpositions, 1

11 indel) and 23 trans (23 transpositions, 13 with inversions) SV-QTLs (Table 12 S5).

14 Consistent with our difficulties in predicting biallelic founder alleles, in 11 SVs

15 the breakpoints were polymorphic among the founders carrying the SV, and in

165 transpositions the orientation of the SV differed between founders. These

17 results emphasise the difficulties frequently reported when delineating SVs. 

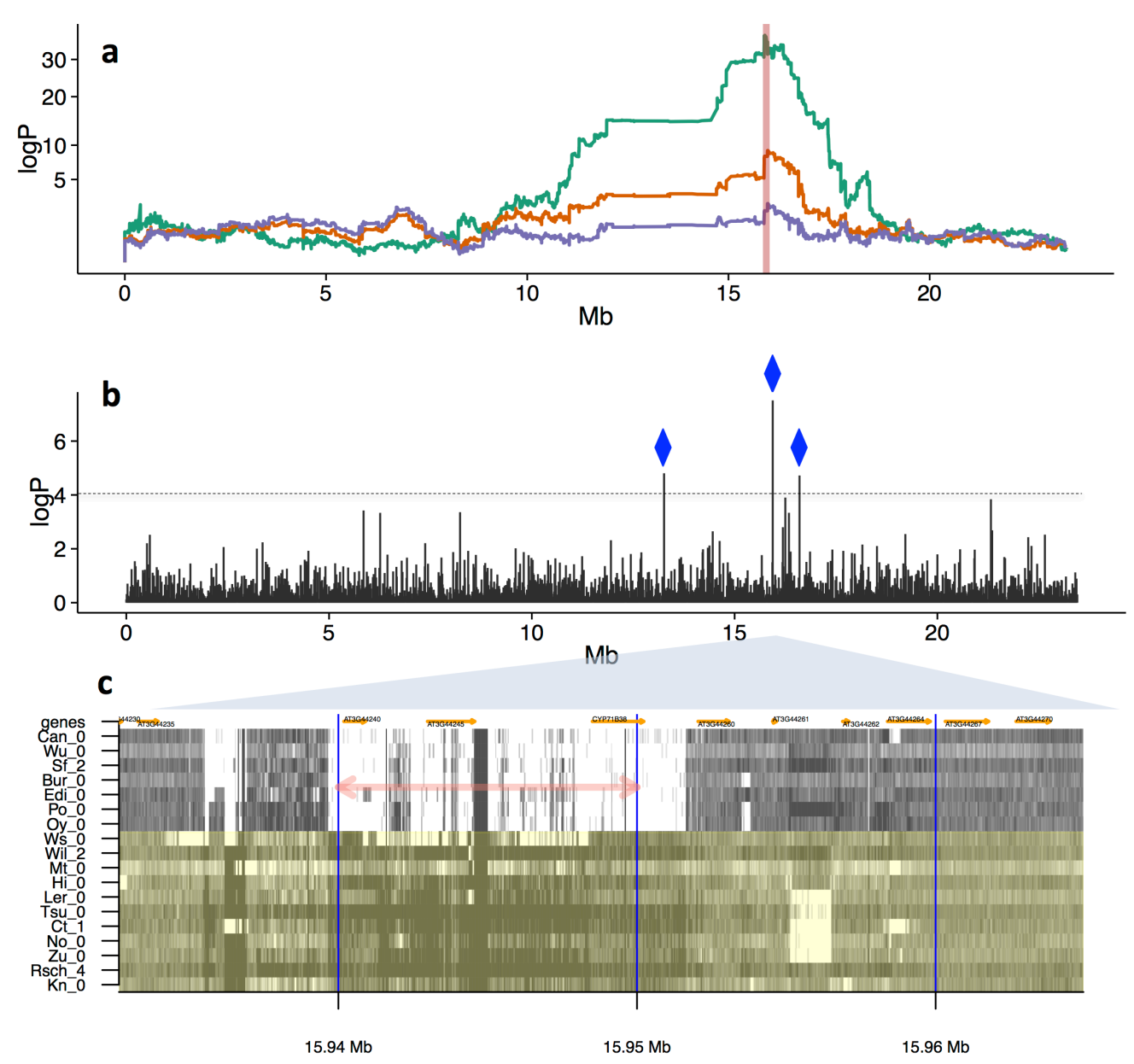

Figure 5 Association of haplotypes and SVs (a) Genome scans over chromosome 3 (x-axis: genomic position, y-axis: $\log \mathrm{P}$ of association). Orange: association of local haplotype with germination time (days), peaking at $15.93 \mathrm{Mb}$. Green: association of local haplotype with the SV trait unpaired reads at the source locus $15.94-15.95 \mathrm{Mb}$ (indicated by the vertical red line), explaining $8.13 \%$ of the variance in germination time, with an SV-QTL mapped at the same position as the germination QTL. Purple: residuals of germination time after regressing out the SV trait, ablating the QTL. (b) Chromosome-wide Pearson correlations between germination time and the numbers of unpaired reads measured at each 10kb source locus (x-axis: genomic position, $y$-axis: $-\log _{10} P$-value of test that the correlation is zero). Three source loci correlate strongly with germination ( $\log P>4$ ), all with cis SVQTLs (blue diamonds). (c) Structural variation in the MAGIC founders. Shown is the read coverage in 18 accessions (labelled on y-axis), over $\sim 30 \mathrm{~kb}$ surrounding around $15.94 \mathrm{Mb}$ (x-axis). Dark shades indicate high coverage, light shades low coverage. The $10 \mathrm{~kb}$ intervals used to define source loci are delineated by vertical blue lines. The source locus giving rise to the SV-QTL in (a), (b) is marked with a pink double-arrow. Those founder accessions predicted to carry the reference allele (No-0, Ct-1, Mt-0, Wil-2, Ler-0, Tsu-0, Rsch-4, Kn-0, Zu-0, Hi-0, Ws-0) are in green, those predicted to carry the SV are in grey. Genes are annotated in orange. 

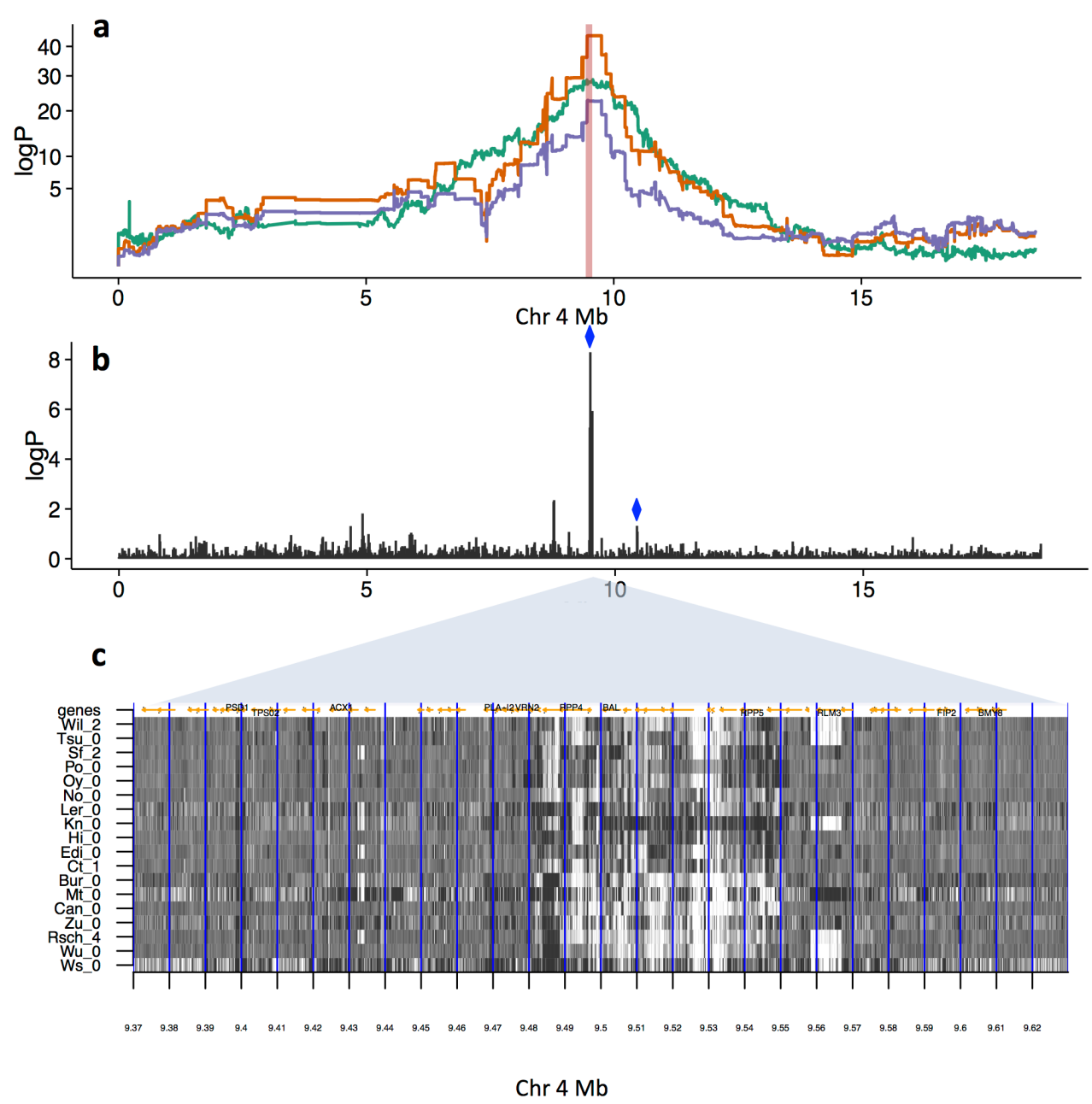

Figure 6 Effects of SVs on resistance to Albugo laibachii infection, (a) Genome scans on chromosome 4. Orange: Association with resistance. The peak of association for is at $9.50 \mathrm{Mb}$. Green: Association with SV-trait improperly paired reads at source 9.50-9.51. Purple: Resistance after two SV traits have been regressed out measuring improperly paired reads (sources chr4, 9.50-9.51Mb (green line) and chr4, 10.44-10.45Mb (not shown), both marked with blue diamonds in fig (b)) that together explain $24.7 \%$ of the phenotypic variance. (b) logP of association between SV traits for improperlypaired reads and the resistance trait. There is a cluster of associated traits near $9.50 \mathrm{Mb}$, in addition to the more weakly associated trait at 10.44 $10.45 \mathrm{Mb}$. (c) Structural variation in high-coverage sequence in the MAGIC founders around $9.50 \mathrm{Mb}$. Shown is the number of improperly-paired reads (dark: high values, light: low values) in 18 accessions (labeled on $y$-axis), between $9.37-9.63 \mathrm{Mb}$ (x-axis). The 10kb intervals used to define source loci are delineated by vertical blue lines. There is a region of complex structural variation spanning $9.48-9.55 \mathrm{Mb}$ approximately, with considerable variation between the founder accessions. Genes are marked by orange arrows, and selected genes, some implicated in disease resistance at this locus, are labelled. 


\section{Effects of SVs on phenotypic QTLs and gene expression}

2 We next investigated associations between SVs and 9 physiological

3 phenotypes, either previously published ${ }^{25,33}$ or new to this study (Table S6).

4 We found 16 distinct SV-QTLs (8 in trans, Table S7) that overlap

5 physiological QTLs. In some cases, regressing the SV-trait from the

6 physiological trait ablated the physiological QTL, consistent with, albeit not

7 proof, that the SV is causal. This is illustrated by a QTL for germination time ${ }^{25}$

8 on chromosome 3, which is ablated by a cis SV-QTL for unpaired reads at

9 around 15,936,650-15,951,640bp (Figure 5a,b). Our analysis predicted that 7

10 founders would carry a deletion at this locus, which was confirmed by the

11 independent founder sequences (Figure 5c), revealing a $15 \mathrm{~kb}$ deletion of

12 three genes, AT3G44240 (Polynucleotidyl transferase, ribonuclease $\mathrm{H}$-like

13 superfamily protein), AT3G44245 (pseudogene of cytochrome P450, family

14 71, subfamily B, polypeptide 21), and CYP71B38 (AT3G44250, cytochrome

15 P450, family 71, subfamily B, polypeptide 38). Other SVs segregate nearby,

16 but with allelic patterns inconsistent with the trait and therefore unlikely to be

17 causal. It is therefore probable that the causal variant(s) lies within the deleted

18 region. The three genes are not known to affect germination, although a

19 mutant of another Polynucleotidyl transferase, AHG2 (AT1G55870) does ${ }^{34}$.

21 We found similar effects on the chromosome 4 QTL for resistance to the

22 fungal pathogen Albugo laibachii, isolate $\mathrm{Nc}^{35}$ (Figure 6, Table S7).

23 Variation in the number of unpaired reads at $9.50-9.51 \mathrm{Mb}$ explains $18.3 \%$ of

24 the variance in resistance, and is adjacent to a cluster of Leucine-rich repeat 
1 genes, and the genes $\mathrm{RPP}^{36}, \mathrm{BAL}^{37}$ and RPP5. This locus is rearranged in

2 some Arabidopsis accessions and known to be involved in disease

3 resistance ${ }^{37}$; Figure 6 confirms the founder genomes have complex,

4 polymorphic SVs in this region. Since the resistance QTL is not completely

5 ablated by the SV traits associated with it, additional non-structural variants

6 likely contribute to it.

8 Importantly, Figures $5 \mathbf{b}, \mathbf{6 b}$ show that correlations between SV traits and

9 phenotypes are tightly localized, generally to width of a single SV trait window,

10 in contrast with wider linkage disequilibrium decay seen in QTL genetic

11 mapping (Figure 5a). Consequently correlations between SV traits and

12 physiological traits can sometimes pinpoint causal variants within

13 physiological QTLs which are otherwise too broad to localize (mapping

14 resolution in the MAGIC population is about $200 \mathrm{~kb}^{25}$ ).

16 We also corroborated studies ${ }^{21,24}$ showing SVs associate with gene 17 dysregulation, even when the gene sequence is undisturbed. Within those 18 SVs with mapped breakpoints, 119 genes spanned the breakpoints, 6,909 lay 19 inside the SVs (Table S8) and 21,747 outside. Using RNA-seq from 200 20 MAGIC aerial seedlings, scaled expression variance increased among genes 21 spanning breakpoints (t-test: $\left.P<9 \times 10^{-3}\right)$ and within SVs $\left(P<1 \times 10^{-13}\right)$

22 (Figure 7a). Similarly larger fractions of lines had silenced transcripts for 23 genes spanning breakpoints (t-test $\left.P<1.2 \times 10^{-2}\right)$ and within SVs $(P<$ 
$12 \times 10^{-52}$ ) (Figure 7b). Expression within SVs was more correlated with local

2 SV traits than outside SVs (F-test $\left.P<2.1 \times 10^{-6}\right)$ (Figure $\left.7 \mathrm{c}\right)$.

a

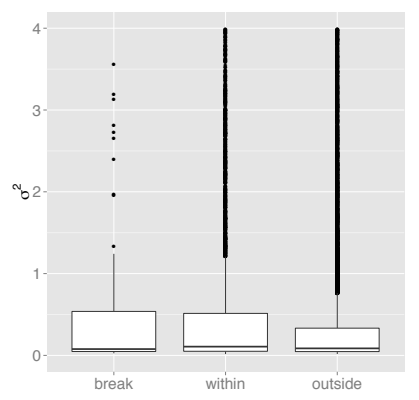

b

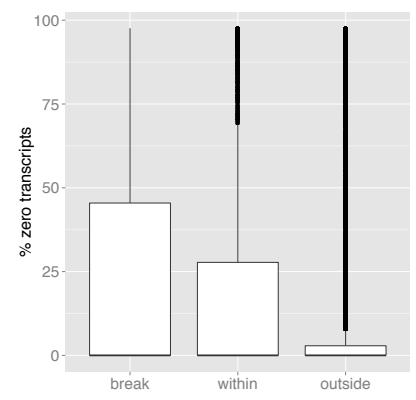

C

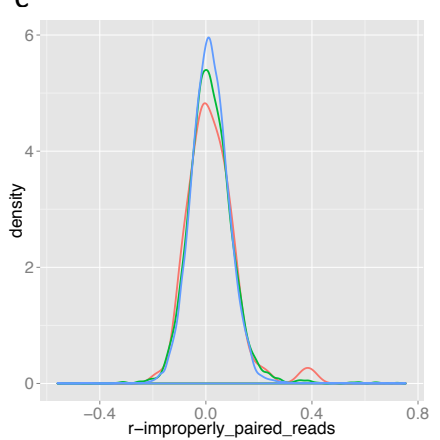

3

$4 \quad$ Figure 7 Variation of expression in 200 MAGIC leaf transcriptomes, in genes 5 spanning SV breakpoints, within SVs or outside SVs. (a) Boxplots of transcript 6 variance (scaled by the mean). (b) Boxplots of the fractions of silenced genes 7 (c) Distributions of the Pearson correlations between gene expression and 8 number of abnormal insert size reads in the locus containing the gene (red: 9 spanning breakpoints, green: within SVs, blue: outside SVs). 


\begin{tabular}{|c|c|c|c|c|c|c|c|}
\hline \multirow[b]{2}{*}{ Phenotype } & \multirow{2}{*}{$h_{H}^{2}$} & \multirow{2}{*}{$h_{S N P}^{2}$} & \multicolumn{5}{|c|}{$\boldsymbol{h}_{S V}^{2}$} \\
\hline & & & IP & LIS & sS & U & U+LIS \\
\hline $\begin{array}{l}\text { Resistance } \\
\text { (resistance to Albugo laibachii) }\end{array}$ & $\begin{array}{l}0.000 \\
(0.139)\end{array}$ & $\begin{array}{l}0.258 \\
(0.085)\end{array}$ & $\begin{array}{l}0.490 \\
(0.335)\end{array}$ & $\begin{array}{l}0.511 \\
(0.307)\end{array}$ & $\begin{array}{l}0.000 \\
(\mathrm{NA})\end{array}$ & $\begin{array}{l}0.673 \\
(0.504)\end{array}$ & $\begin{array}{l}0.503 \\
(0.314)\end{array}$ \\
\hline $\begin{array}{l}\text { RosetteLeafNumber.LongDay } \\
\text { (number of leaves in a rosette for } \\
\text { plants grown under long daylight }\end{array}$ & $\begin{array}{l}0.228 \\
(0.081)\end{array}$ & $\begin{array}{l}0.322 \\
(0.076)\end{array}$ & $\begin{array}{l}0.463 \\
(0.148)\end{array}$ & $\begin{array}{l}0.456 \\
(0.146)\end{array}$ & $\begin{array}{l}1.000 \\
(\mathrm{NA})\end{array}$ & $\begin{array}{l}1.000 \\
(0.377)\end{array}$ & $\begin{array}{l}0.447 \\
(0.146)\end{array}$ \\
\hline $\begin{array}{l}\text { RosetteLeafNumber.ShortDay } \\
\text { (number of leaves in a rosette for } \\
\text { plants grown under short daylight) }\end{array}$ & $\begin{array}{l}0.038 \\
(0.060)\end{array}$ & $\begin{array}{l}0.047 \\
(0.062)\end{array}$ & $\begin{array}{l}0.000 \\
(\mathrm{NA})\end{array}$ & $\begin{array}{l}0.000 \\
(\mathrm{NA})\end{array}$ & $\begin{array}{l}0.000 \\
(\mathrm{NA})\end{array}$ & $\begin{array}{l}0.000 \\
(\mathrm{NA})\end{array}$ & $\begin{array}{l}0.000 \\
(\mathrm{NA})\end{array}$ \\
\hline $\begin{array}{l}\text { bolting.Bath } \\
\text { (bolting time in a greenhouse) }\end{array}$ & $\begin{array}{l}0.426 \\
(0.064) \\
\end{array}$ & $\begin{array}{l}0.476 \\
(0.048) \\
\end{array}$ & $\begin{array}{l}0.783 \\
(0.093) \\
\end{array}$ & $\begin{array}{l}0.783 \\
(0.093) \\
\end{array}$ & $\begin{array}{l}0.952 \\
(0.047) \\
\end{array}$ & $\begin{array}{l}0.989 \\
(0.025) \\
\end{array}$ & $\begin{array}{l}0.785 \\
(0.092) \\
\end{array}$ \\
\hline $\begin{array}{l}\text { days.to.germ.x } \\
\text { (germination time) }\end{array}$ & $\begin{array}{l}0.220 \\
(0.068)\end{array}$ & $\begin{array}{l}0.149 \\
(0.063)\end{array}$ & $\begin{array}{l}0.385 \\
(0.116)\end{array}$ & $\begin{array}{l}0.357 \\
(0.113)\end{array}$ & $\begin{array}{l}0.598 \\
(0.165)\end{array}$ & $\begin{array}{l}0.835 \\
(0.146)\end{array}$ & $\begin{array}{l}0.365 \\
(0.114)\end{array}$ \\
\hline $\begin{array}{l}\text { fieldFT.pl } \\
\text { (flowering time in the field) }\end{array}$ & $\begin{array}{l}0.000 \\
(0.068) \\
\end{array}$ & $\begin{array}{l}0.095 \\
(0.076) \\
\end{array}$ & $\begin{array}{l}0.000 \\
(0.179) \\
\end{array}$ & $\begin{array}{l}0.000 \\
(0.130) \\
\end{array}$ & $\begin{array}{l}0.000 \\
(0.913) \\
\end{array}$ & $\begin{array}{l}0.000 \\
(\mathrm{NA})\end{array}$ & $\begin{array}{l}0.000 \\
(0.145) \\
\end{array}$ \\
\hline $\begin{array}{l}\text { fieldRD.pl } \\
\text { (rosette diameter plasticity) }\end{array}$ & $\begin{array}{l}0.000 \\
(\mathrm{NA})\end{array}$ & $\begin{array}{l}0.000 \\
(0.063)\end{array}$ & $\begin{array}{l}0.000 \\
(0.085)\end{array}$ & $\begin{array}{l}0.000 \\
(0.084)\end{array}$ & $\begin{array}{l}0.000 \\
(0.239)\end{array}$ & $\begin{array}{l}0.166 \\
(0.220)\end{array}$ & $\begin{array}{l}0.000 \\
(0.085)\end{array}$ \\
\hline $\begin{array}{l}\text { leaves.day.28.given.days.to.germ } \\
\text { (residuals for number of leaves at } \\
\text { day28 regressed on germination) }\end{array}$ & $\begin{array}{l}0.193 \\
(0.081)\end{array}$ & $\begin{array}{l}0.299 \\
(0.066)\end{array}$ & $\begin{array}{l}0.391 \\
(0.146)\end{array}$ & $\begin{array}{l}0.362 \\
(0.140)\end{array}$ & $\begin{array}{l}0.836 \\
(0.189)\end{array}$ & $\begin{array}{l}0.675 \\
(0.272)\end{array}$ & $\begin{array}{l}0.366 \\
(0.142)\end{array}$ \\
\hline $\begin{array}{l}\text { ttl_branch.BATH } \\
\text { (total number of branches of plants) }\end{array}$ & $\begin{array}{l}0.106 \\
(0.048)\end{array}$ & $\begin{array}{l}0.196 \\
(0.054)\end{array}$ & $\begin{array}{l}0.276 \\
(0.104)\end{array}$ & $\begin{array}{l}0.275 \\
(0.100)\end{array}$ & $\begin{array}{l}0.419 \\
(0.193)\end{array}$ & $\begin{array}{l}0.616 \\
(0.214)\end{array}$ & $\begin{array}{l}0.279 \\
(0.102)\end{array}$ \\
\hline
\end{tabular}

1

2 Table 2 Estimates of heritability. $\boldsymbol{h}_{H}^{2}$ is haplotype-based heritability. $\boldsymbol{h}_{S N P}^{2}$ is 3 SNP-based heritability. $\boldsymbol{h}_{\boldsymbol{S} V}^{2}$ is the heritability estimated from structural variant 4 anomaly traits. Numbers in brackets are the standard errors of the heritability 5 estimates above. ER: Excess Reads, IP: Improperly-paired, LIS: Large Insert 6 Size, SS: Same Strand, U: Unpaired, U+LIS: Unpaired or Large Insert Size. 7 Heritabilities for excess reads are not reported because the fraction of bins in 8 any individual containing non-zero entries was too small.

Effects of SV-traits on Heritability

12 Finally, we treated the SV traits as if they were quantitative noisy genotypes to

13 define pair-wise correlations between MAGIC lines, as weighted correlations

14 of their SV traits (Methods). We constructed SV genetic relationship matrices

15 (GRMs) $K_{S V}$, which we used to compute the SV-heritability $h_{S V}^{2}$ of each of the

16 physiological traits mapped above by analogy with the mixed models used for 
1 estimating SNP-based heritability ${ }^{38}$. This idea is similar to the use of gene

2 expression data to model intersample relationships ${ }^{39}$. We also compared

3 these SV-heritabilities with those obtained from "classical" haplotype $K_{H}$ or

4 SNP-based $K_{S N P}$ GRMs (Table 2). $K_{H}$ was computed from the identity

5 between haplotype mosaics (and so measures identity by descent), $K_{S N P}$ and

$6 K_{S V}$ were computed from the correlations of $1.2 \mathrm{M}$ imputed SNPs or $12 \mathrm{k}$ SV-

7 traits respectively (Methods). We also computed SV heritability when only the

8 most variable $50 \%$ or $25 \%$ of SV-traits were included, to investigate if

9 heritability was concentrated at the most structurally variable loci.

11 As expected, SNP-based heritability $h_{S N P}^{2}$ is generally similar to haplotype-

12 based heritability $h_{H}^{2}$ for all phenotypes tested. However, the heritability $h_{S V}^{2}$

13 captured by the six measures of SV anomaly is more variable, sometimes 14 being close to zero, but sometimes exceeding classical heritability by a 15 considerable margin (Table 2). The standard error of $h_{S V}^{2}$ was typically about 16 twice that of $h_{S N P}^{2}$ or $h_{H}^{2}$, (approximately 0.1 compared to 0.05 ), presumably 17 reflecting greater uncertainty in SV-traits than in SNPs or haplotypes. 18 Therefore the larger heritability estimates should be treated with caution. 19 Nonetheless, for phenotypes such as times for germination or bolting, the 20 standard errors of all estimates are comparable at $\sim 0.05$ and is possible to 21 make meaningful comparisons. Figure 8A,B illustrates likelihood curves the 22 times to germination $(\mathrm{A})$ bolting $(\mathrm{B})$, for SNP, haplotype and large insert-size 23 anomalies. Visualising the entire curves gives a better sense of the 24 uncertainty of the maximum likelihood estimates at the curves' minima (the 
1 standard errors in Table 2 are asymptotic estimates based on the curvature at

2 these minima). The Figure $8 \mathrm{~B}$ shows that for bolting time the heritability

3 attributable to all largeisize SV-traits, $h_{\text {largeisize }}^{2}$, is close to $80 \%$, compared to

$4 \quad 40-50 \%$ for haplotype or SNP-based estimates. As the fraction of SV traits is

5 reduced by progressively removing those traits with lower variance, $h_{\text {largeisize }}^{2}$

6 reduces to that of SNPs or haplotypes. This suggests that there is genome-

7 wide structural variation that is not tagged by standard genetic variation, and

8 which has important effects on specific phenotypes. These effects are not

9 universal, as Figure $8 \mathrm{~A}$ shows for germination time, where heritability is

10 similar for all estimates.

11

12 The relative independence of the heritability estimates borne out by low

13 correlations between the corresponding elements of SNP and SV-based

14 GRMs, which range around 0.3 depending on the anomaly type (Figure 8D

15 shows the relationship between GRMs computed from SNPs vs large

16 insertsize anomalies), compared to the correlation of 0.93 between SNP and

17 haplotype based GRMs (Figure 8C). 
A

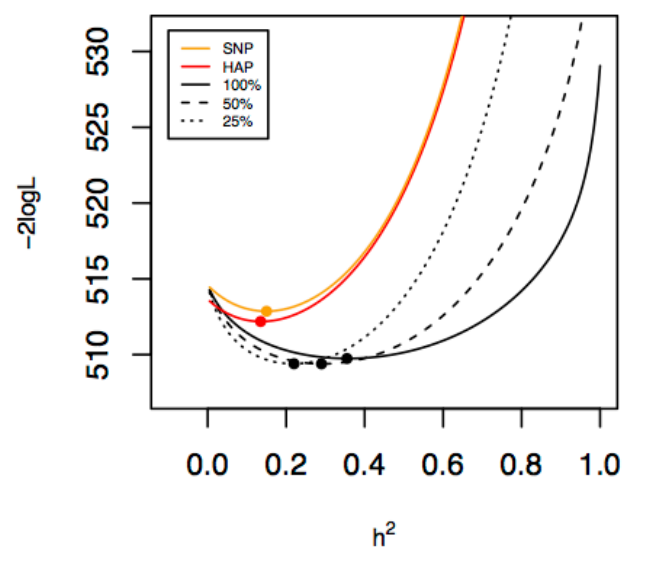

C

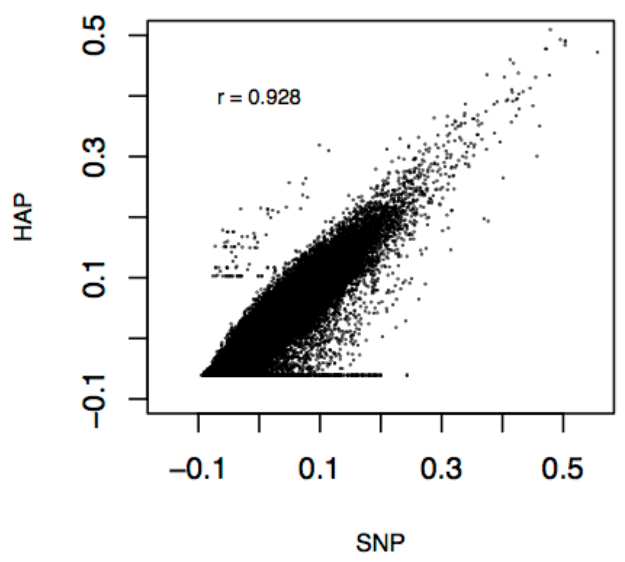

B

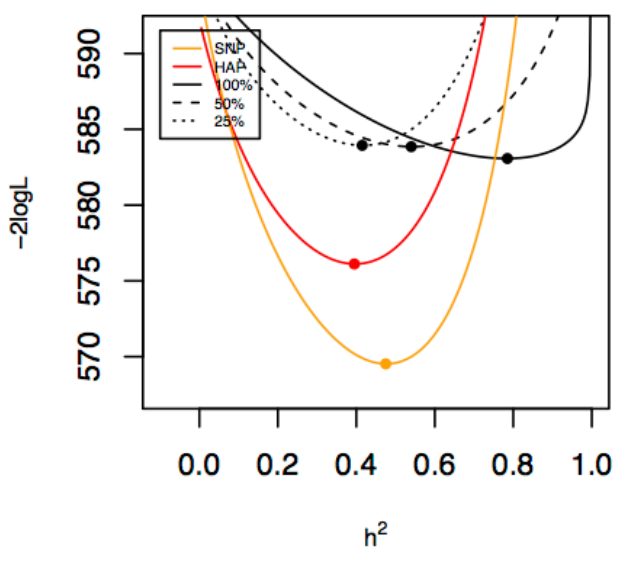

D

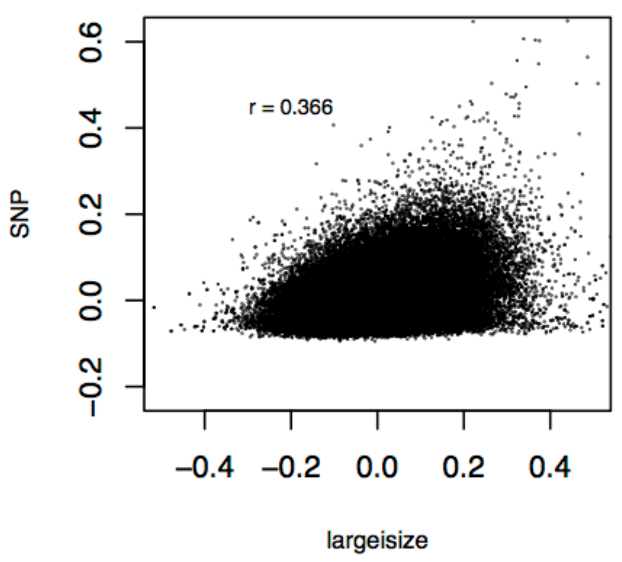

2 Figure $8(a, b)$ log-likelihood curves for two phenotypes bolting.BATH (large 3 insert size read anomalies) and RosetteLeafNumber.ShortDay (unmapped 4 read anomalies), illustrating contrasting behavior of heritability estimates 5 based on structural variants, SNPs and haplotypes. Log-likelihood curves as 6 functions of heritability are plotted for the GRMs estimated from SNPs, 7 haplotypes and various fractions of anomalies. The maximum likelihood 8 estimates of each heritability measure correspond to the minima of the 9 corresponding curves, and are marked with dots. (c,d) Scatter plots 10 comparing the off-diagonal elements of genetic relationship matrices. (c) $K_{S N P}$ vs $K_{H} ;$ (d) $K_{S N P}$ vs $K_{\text {largeisize }}$. 
Discussion

3 Our aim has been to understand better the architecture and impact of

4 structural variation in populations sequenced at low coverage. We used a

5 strategy that combines analysis of read-mapping signatures commonly used

6 to detect SVs in individuals sequenced at high coverage, with association

7 mapping in populations ${ }^{40}$. A somewhat related concept was used for mapping

8 un-localised contigs into reference assemblies based on linkage

9 disequilibrium ${ }^{41}$.

11 In doing so, we have generated a partial catalog of SVs in Arabidopsis,

12 although our purpose is not to call SVs systematically, a task that remains

13 challenging with short reads. Rather, we have shown how SVs' impact can be 14 assayed without necessarily calling them or mapping their breakpoints. In this 15 way, we can distinguish transpositions from local SVs, and determine the 16 approximate locations of transpositions. The privileged role of the reference 17 genome in the analysis means that some transpositions appear as deletions, 18 so we probably have underestimated their true frequency. Despite this, a 19 quarter of the SVs we detected are transpositions. Given the large numbers of 20 transposable elements in Arabidopsis - over 11,000 from over 300 families

21 are annotated in the reference ${ }^{24}$ - this is unsurprising. However, many of the 22 SVs we mapped are too large, covering tens of kilobases, to be single 23 transposon-mediated events. 
1 In the minority of cases where we delineated breakpoints exactly, we often

2 found SVs are complex combinations of different SV types. But often

3 breakpoints were not simple cut-and-paste transformations of the reference

4 genome, as illustrated in Figure 6c. Indeed, it is impossible to determine

5 precisely the changes that led to many observed structural variants.

7 Because we used ultra-low-coverage $0.3 x$ sequence data, we divided the

8 Arabidopsis genome into $10 \mathrm{~kb}$ bins when counting read-mapping anomalies.

9 With higher coverage and a larger sample size it would be possible to use a

10 larger number of narrower bins, thereby improving resolution. The public

11 release of over 3000 rice genomes sequenced at $\sim 14 x^{42}$ and over 1000

12 Arabidopsis accessions sequenced at over $\sim 20 x^{43}$ means that there are now

13 large collections of inbred plant genomes available for analysis. Both of these

14 sets are worldwide surveys of germplasm, in which we expect SVs to

15 contribute significantly to, and be confounded with, their extensive population

16 structure, in contrast to the MAGIC population used here. Disentangling these

17 effects will be a challenging but important task.

19 Mapping SVs in a population brings new insights to the problem of QTL 20 analysis. First, an SV trait inside a QTL may entirely explain the genetic effect 21 at the QTL, and hence provide support for being the causal variant (e.g.

22 Figure 6). Second, SV traits are much more tightly localized than are QTLs:

23 there is little or no correlation between neighbouring SV traits so there are no 24 effects of linkage disequilibrium. Our analysis also shows that expression of 
1 genes is often dysregulated or even silenced within large SVs, raising the

2 possibility that an SV causes multiple regulatory and phenotypic effects.

3

4 Finally we have shown that even in a population like Arabidopsis MAGIC

5 where the local haplotype space is known, structural variation has an impact

6 on heritability that cannot be explained by standard genetic variation. This is

7 unexpected given the breeding history and genetic architecture of the MAGIC

8 lines. For if an SV segregated among the founders of the MAGIC lines, then it

9 should be tagged by the local haplotype context, and therefore contribute to 10 both $h_{H}^{2}$ and $h_{S V}^{2}$.

12 One possible explanation is that structural variation at loci rich in mobile

13 elements accumulates within each lineage, leading to SVs that are private to 14 each MAGIC line but tend to occur at the same loci, thereby creating similar 15 phenotypic effects. Supporting this, in our analysis the SV-relationship matrix 16 is calculated empirically, without regard to the ancestry of the MAGIC lines, 17 being solely a function of the counts of read-mapping anomalies. Therefore, 18 recalling that the history of each MAGIC line includes a private lineage of at 19 least five generations of selfing, should SVs accumulate recurrently but 20 independently in different lineages, then these could generate phenotypic 21 associations invisible to SNP or haplotype variation. In Arabidopsis, it is 22 known that some mobile elements are methylated, often in response to 23 environmental cues, and that such methylation plays a role in the epigenetic 24 control of certain phenotypes ${ }^{44}$. Testing this hypothesis in Arabidopsis MAGIC 
1 lines would require complete and precise reassembly of each genome using

2 long reads, annotation of mobile elements and determination of their

3 methylation status.

4

5 The general role that recurrent, but independent, genomic rearrangements

6 might play in Arabidopsis and in other species remains to be seen, but there

7 is no a priori reason why it should not be a driver of phenotypic variation. The

8 approach used here may therefore have wider application to other

9 populations, both to characterize the extent of transpositions and the impact of

10 cryptic structural variation on phenotypes.

11 


\section{Methods}

3 DNA extraction and sequencing MAGIC lines were grown at Bath (lab of $P$.

4 Kover) or Oxford (lab of N.P. Harberd) in greenhouses or growth chambers

5 respectively. Leaves were harvested for DNA extraction. DNA isolation was

6 performed at the John Innes Centre, in 96 well plates using the DNeasy 96

7 Plant Kit and DNeasy 96 Protocol (www.quiagen.com). Sequencing was

8 performed by the Oxford Genomics Centre.

9

10 Genomic DNA library construction and multiplexing Samples were

11 quantified using the Quant-iT ${ }^{\text {тм }}$ PicoGreen ${ }^{\circledR}$ dsDNA Kits (Invitrogen) and a

12 Genios plate scanner (Tecan) according to manufacturer specifications.

13 Sample integrity was assessed using $1 \%$ agarose gel. Approximately $300 \mathrm{ng}$ 14 of DNA were fragmented using a Covaris S2 system with the following 15 settings: Intensity: 5, Duty Cycle: 20, Cycles per Burst: 200, Time: 60 sec. 16 Distribution of fragments after shearing was determined using a Tapestation 17 D1200 system (Agilent/Lab901). DNA Libraries were constructed using the 18 NEBNext DNA Sample Prep Master Mix Set 1 Kit (NEB) with minor 19 modifications and a custom automated protocol on a Biomek FX (Beckman).

20 Ligation of adapters was performed using Illumina Adapters (Multiplexing 21 Sample Preparation Oliogonucleotide Kit). Ligated libraries were size selected 22 using Ampure magnetic beads (Agencourt). Each library was PCR enriched 23 with $25 \mu \mathrm{M}$ each of the following custom primers:

24 Multiplex PCR primer 1.0 
15

2 AATGATACGGCGACCACCGAGATCTACACTCTTTCCCTACACGACGCTCT

3 TCCGATCT-3'

$4 \quad$ Index primer

$5 \quad 5$

6 CAAGCAGAAGACGGCATACGAGAT[INDEX]CAGTGACTGGAGTTCAGACG

7 TGTGCTCTTCCGATCT-3'

8 Indexes used were 8bp long (manuscript in preparation). Enrichment and

9 adapter extension of each preparation was obtained using $5 \mu$ l of size selected

10 library in a $50 \mu \mathrm{l}$ PCR reaction. After 10 cycles of amplification (cycling

11 conditions as per Illumina recommendations) the reactions were purified with

12 Ampure beads (Agencourt/Beckman). The final size distribution was

13 determined using a Tapestation 1DK system (Agilent/Lab901). The

14 concentrations used to generate the multiplex pool were determined by

15 Picogreen. The library resulting from the pooling was quantified using the 16 Agilent qPCR Library Quantification Kit and a MX3005P instrument (Agilent)

17 before sequencing on an Illumina GAllx as 50bp or 100bp paired end reads.

18 All steps for library construction, including the setup of the PCR reaction were

19 performed on a Biomek FX (Beckman). Post PCR cleanup was carried out on

20 a Biomek NXp (Beckman) whereas a Biomek 3000 (Beckman) was used to

21 generate the pools of 96 indexed libraries.

23 Processing Sequence Reads and SNP Calling The Illumina reads were

24 mapped to the $A$ thaliana reference genome (TAIR10) using Stampy version 
1 v1.0.20 ${ }^{25}$. Alignments were stored in a separate BAM file for each MAGIC

2 line. Previous sequencing for the 18 MAGIC line progenitors had produced a

3 catalogue of $3,316,270$ segregating $\mathrm{SNPs}^{17}$. We ran GATK $\mathrm{v} 2.6^{26}$ on the

4 segregating SNPs to call variants for the 19 founders, setting the following

5 read filters: Allele Balance, BaseQualityRankSumTest, Clipping

6 RankSumTest, Coverage, DepthPerAlleleBySample, FisherStrand,

7 GCContent, HaplotypeScore, LowMQ, MappingQualityRankSumTest,

8 MappingQualityZero, MappingQualityZeroBySample, RMSMappingQuality,

9 ReadPosRankSumTest. We filtered out SNPs that were triallelic, within

10 transposons, or heterozygous for any founders.

13 Definition of Structural Variant Traits We divided the TAIR10 (Col-0)

14 reference genome into 11,915 abutting $10 \mathrm{~kb}$ segments. Within each segment

15 we computed six measures of anomalously mapped reads that are signatures

16 of SVs. Let $R$ be the set of all reads mapped to a genome of length $L ; \rho$ is the

17 number of reads in $R$, and $\rho_{l}$ the number of reads mapped to a segment $l$ of

18 length $10 \mathrm{~kb}$. The read anomaly measures computed in each segment are:

19 1. High read coverage: $\rho_{h c}=\rho_{l}-1.5 E\left[\rho_{l}\right]$, where $E\left[\rho_{l}\right]=\frac{\rho \times l}{L}$ is the 20 expected read coverage of the segment

212 Unpaired reads: $\boldsymbol{\rho}_{\boldsymbol{u}}$ number of reads mapping to the segment whose 22 pair is not mapped

23 3. Pairs on the same strand: $\rho_{\boldsymbol{s}}$ number of reads with pair on the same 24 strand 
1 4. Reads with large insert size: $\rho_{i s}$ number of read pairs with insert size

2 outside the range $m_{s} \pm I Q R_{S}$ or mapped to different chromosomes,

$3 m_{s}, I Q R_{s}$ being the median and interquartile range of insert sizes of all the

4 reads in the sample.

5 5. Unpaired reads or with large insert size: $\rho_{u i}=\rho_{u}+\rho_{i}$

6 6. Improperly paired reads: $\rho_{u i s}=\rho_{u}+\rho_{i}+\rho_{s}$

7 The last two traits are combination of others - certain SV types can cause

8 multiple anomaly signatures, so merging them may increase power. Each type

9 of read pair anomaly was measured in each of the $11,91510 \mathrm{~kb}$ segments,

10 determining 71,490 traits in total.

11 Genome scan We treated the SV traits like a gene expression eQTL study, 12 performing a genome scan for each one. Association was tested by fitting trait

13 vectors to the imputed ancestral haplotype at each locus in the 488 genome 14 mosaics. In combination, the mosaics partitioned the genome into 16,700 15 haplotype blocks, with the ancestral haplotype of all lines unchanged in each 16 one. Let $y_{A i}$ be the number of anomalous reads of a certain type at source 17 segment $A$ in line $i$. At every haplotype block $p$ we fit the linear model:

$$
y_{A i}=\mu_{A}+\sum_{s \in S} X_{p i}(s) \beta_{A p}(s)+e_{i}
$$

$18 \mu_{A}$ is the average trait value at $A, X_{p i}(s)$ is a binary indicator of whether line $i$

19 carries haplotype $s$ at $p, \beta_{A p}(s)$ is the effect of founder haplotype $s$ and $e_{i}$ the 20 (Normally distributed) error. The founder effects $\beta_{A p}(s)$ were estimated by an 21 one-way ANOVA with null hypothesis: 


$$
H_{0}(p): \beta_{A p}(s)=0 \forall s
$$

1 Genome-wide significance We denote the location of a sink locus

2 associated with an SV trait an SV-QTL. Each genome scan of a given SV trait

3 returns a $p$-value $\pi_{A p}$ for each of the 16,700 scanned blocks $p$. We selected

4 as candidate SV-QTLs for each mapped trai the locus with maximum

5 genome-wide negative logarithm of $\pi_{A p}$, i.e.

$$
\lambda_{A}=\max \left(-\log _{10}\left(\pi_{A p}\right)\right)
$$

6 To control for the number of test in each scan $(16,700)$ and correct for

7 associations driven by outliers we performed 100 permutations $T_{A}$ of the trait

8 vector $y_{A i}$ and repeated the mapping for each one. We then fitted a

9 generalized extreme value distribution (GEV), using $R$ evd package on the

$10 \lambda_{A}(t), t \in T_{A}$ values obtained by permutation, from which we obtained a

11 genomewide corrected p-value:

$$
\gamma_{A}=-\log \left(1-\exp \left(1+\hat{s}_{A}\left(\frac{\lambda_{A}-\hat{\alpha}_{A}}{\hat{b}_{A}}\right)^{-\frac{1}{\hat{s}_{A}}}\right)\right.
$$

$12 \hat{a}_{A}, \hat{b}_{A}, \hat{s}_{A}$ are the MLEs of $a_{A}, b_{A}, s_{A}$. Study-wide SV-QTLs are selected at $13 F D R<10^{-2}\left(\gamma_{A}<10^{-3}\right)$. At this FDR we mapped 10,275 SV-QTLs in total.

14 Table S3 shows mapped QTLs per read anomaly category. 3,773 SV-QTLS

15 had coincident sources and sinks, probably corresponding to the same SV, 16 and so were counted only once. SVs are tabulated in Table S4.

17 Cis and trans SV-QTLs In total we mapped 6,502 distinct SV-QTLs, each 
1 corresponding to a unique SV. SV-QTLs with sources and sinks within $2 \mathrm{Mb}$

2 from each other were classified as cis, and the rest as trans.

3 Prediction of SV allele frequency We predicted the founder haplotypes

4 carrying SVs at the origination of the population, using the fact that SV

5 haplotypes will have elevated anomalous reads at the source. For each SV-

6 QTL the founders' contributions were arranged as a $19 \times 19$ table $T$ whose

7 cells $(i, j)$ carry the sum of read anomalies (of a certain type) at the source for

8 all lines carrying haplotype $i$ at the sink and haplotype $j$ at the source. A

9 founder is classified as carrying the SV if its corresponding row has generally

10 higher values than the rest of the table (we note that in cis QTLs the matrix is

11 almost diagonal).

12 For each cell of $T$, let $t_{i j}$ be the sum of trait values for all genomes carrying

13 haplotypes $i, j$ at the sink and source, respectively. The contributions of

14 founder $i$ are estimated as the "row" effect: $r_{i}=\sum_{j} t_{i j}$, which are reorder such

15 that $r_{1}>\cdots>r_{19}$, with $r_{1} \approx \cdots \approx r_{19}$ under null. We rejected the null hypothesis

16 if there is a set $\left\{r_{1}, \ldots r_{k}\right\}$ such that $r_{1}>\cdots>r_{k}>r_{k+1}>\cdots>r_{19}$. There are 18

17 such possible sets. The z-score of each set $k$ is:

$$
z_{k}=\frac{\sum_{j=1}^{k} r_{j}-E\left[r_{k}\right]}{\sigma\left(r_{k}\right)}
$$

$18 E\left[r_{k}\right]$ and $\sigma\left(r_{k}\right)$ are estimated by 1000 permutations of $T$, denoted as $R_{z_{k}}$. We

19 choose $k$ such that $z_{k}$ is maximised. $k$ is significant, hence the corresponding

20 founders carry the SV if the permutation p-value $\pi_{z_{k}}=\frac{\left|r_{z_{k}} \in R_{z_{k}}: r_{z_{k}} \geq z_{k}\right|}{N} \leq 10^{-2}$. 
1 Examples of cis and trans SV-QTL tables with detectable founders are shown

2 in Figure S1. The test predicts founder haplotype groups at 2,391 SV-QTLs.

\section{Heritability}

4 For a given phenotype (such as germination time) measured in the MAGIC

5 lines, the phenotypic variance matrix is represented by the mixed model

$6 \quad V=K \sigma_{g}^{2}+I \sigma_{e}^{2}$ where $K$ is the genetic relationship matrix (GRM) and $I$ the

$7 \quad$ identity matrix. The phenotypic heritability is

8

$$
h^{2}=\sigma_{g}^{2} /\left(\sigma_{g}^{2}+\sigma_{e}^{2}\right)
$$

11 We computed genetic relationship matrices $K$ between the inbred MAGIC

12 lines in three ways:

Identity By Descent (haplotype-based) $K_{H}$ We used the representations of

15 chromosomes as homozygous mosaics of the 19 founders to determine

16 identity by descent (IBD). Across all $N$ MAGIC lines, we identified the union of

17 the mosaic breakpoints, and then segmented the genome of each MAGIC line according to these breakpoints. Thus by construction, the founder haplotype

19 for each line is constant within each segment. The founder haplotype in

20 segment $L$ in line $i$ is represented by an indicator matrix $H_{i L f}$ which is 1 if the

21 founder is $f$ and 0 otherwise. If $w_{L}$ is the fraction of the genome covered by $L$, 
1 and $f_{i j L}=\sum_{f} H_{i L f} H_{j L f}$ is the indicator of whether lines $i, j$ are IBD at $L$, then

2 the fraction of the genome that is IBD for lines $i, j$ is

3

$$
d_{i j}=\sum_{L} w_{L} f_{i j L}
$$

4

5 This matrix is then standardised to take the form of a genetic relationship

6 matrix. Let $P_{L}$ be the probability that, given the observed population-wide

7 founder haplotype fractions at $L$, two randomly-sampled lines are IBD, i.e.

8

$$
P_{L}=2 \sum_{i<j} f_{i j L} / N(N-1)
$$

9

10

11 Define

12

$$
\begin{gathered}
E_{i j}=\sum_{L} w_{L}\left(f_{i j L}-P_{L}\right) \\
\mu_{i}=\sum_{j} E_{i j} / N \\
\sigma_{i}^{2}=\sum_{j}\left(E_{i j}^{2} / N\right)-\mu_{i}^{2}
\end{gathered}
$$

13

14

15 in order to compute the standardised IBD matrix $\boldsymbol{K}_{\boldsymbol{H}}$ 


$$
K_{H i j}=\frac{\sum_{L} w_{L}\left(f_{i j L}-\mu_{L}\right)}{\sigma_{i} \sigma_{j}}
$$

1 which has main diagonal 1 and off diagonal elements in the range $[-1,1]$.

2

3 Identity by State (SNP-based) $\boldsymbol{K}_{\boldsymbol{S}}$ SNPs were imputed in the MAGIC lines by

4 using the haplotype mosaics and the catalog of variants in the 19 founders.

5 We treated each MAGIC line as being homozygous. We investigated down-

6 sampling the number of SNPs (as the total is over 1 million). Subsamples of

7 between $1 \%$ and $10 \%$ of the total SNPs were used to define the GRM in the

8 usual way for a homozygous population. Thus if $S_{i p} \in\{0,1\}$ encodes the SNP

9 genotype in individual $i$ and SNP $p$, and if $\pi_{p}$ is the allele frequency at $p$, then

10 the normalized genotype is

$$
T_{i p}=\frac{S_{i p}-\pi_{p}}{\sqrt{\pi_{p}\left(1-\pi_{p}\right)}}
$$

11

12 Since the MAGIC lines are almost fully inbred the normalization is different

13 from that in an outbred population under Hardy-Weinberg equilibrium. The

14 SNP-based GRM is the matrix $\boldsymbol{K}_{\boldsymbol{S}}$ with elements

15

$$
K_{S i j}=\sum_{p} T_{i p} T_{j p} / M
$$

16

17 That is,

$$
K_{S}=\boldsymbol{T} \boldsymbol{T}^{\prime} / M
$$


1 which is always positive semi-definite.

2

3 Read Anomalies $K_{R}$ We constructed read-anomaly GRMs by analogy to

4 SNP-based GRMs. Let $X_{i L}$ be the read anomaly trait for individual $i$ at locus $L$.

5 Let $\alpha_{L}$ and $\tau_{L}^{2}$ be the sample means and variances:

$$
\alpha_{L}=\sum_{i} X_{i L} / N
$$

6

$$
\tau_{L}^{2}=\sum_{i} X_{i L}^{2} / N-\alpha_{i}^{2}
$$

7

8 Define the standardized trait matrix $\boldsymbol{W}$ with elements

9

$$
W_{i L}=\frac{\left(X_{i L}-\alpha_{L}\right)}{\tau_{L}}
$$

11 The genetic relationship between individuals $i, j$ is

$$
K_{R i j}=\sum_{L} W_{i L} W_{j L} / M
$$

14 where $M$ is the number of loci. Then the read anomaly GRM $K_{R}=\boldsymbol{W} \boldsymbol{W}^{\prime} / M$.

16 This formulation guarantees that the GRM is positive. The choice of loci that

17 contribute to the GRM can be varied. Loci at which there is no variation in

18 read anomaly are superfluous and so are ignored. Similarly, loci in which only 
1 a small fraction of individuals are anomalous (say <3\%) are likely to carry too

2 much weight after normalization and so may optionally be dropped,

3 analogously to the calculation of SNP-based GRMs using only high-frequency

4 SNPS.

5

6 In the MAGIC population, each of the 19 founders should be present at a

7 given locus in about $1 / 19=5.5 \%$ of lines. Thus an SV that is private to a

8 single founder should give rise to a trait which is null (ie its un-normalised trait

9 value is zero) in $94.5 \%$ of lines on average. Loci with a much smaller fraction

10 of non-null trait values might represent private structural mutations.

We computed a separate kinship matrix for each of the six measures of read anomaly, and estimated heritability by maximum likelihood.

Validation by paired-end data We used high and low coverage paired-end

16 reads from the 19 founders $^{8}$ and from the MAGIC lines to search for

17 enrichment of read pairs linking the source and sink. For the high-coverage

18 test we restricted attention to the 2,391 SV-QTLs in which founders carrying

19 the SV are predicted, and compared the number of read pairs with one read

20 mapped in the $10 \mathrm{~kb}$ of the source and within a variably-sized window of $W \mathrm{~kb}$

21 from the sink (association peak) ( $W \in\{5,20,30,40,50,100,150,200,400\})$ in the

22 founders carrying the SV to the remaining founders using two-sided Fisher's

23 exact tests (FET) at the 5\% level of significance. Given the haplotype

24 structure of the MAGIC lines, mapping resolution is variable between QTLs in 
1 MAGIC - (200kb being the average in MAGIC $\left.{ }^{25}\right)$ and may depend on the

2 significance of the association. In the low coverage data we performed the

3 same test comparing the 100 lines with the highest read anomaly trait value to

4 the rest of the population.

5 Validation by denovo contigs We used BLAT ${ }^{45}$ to align $5,524,143$ short

6 contigs (50-1000bp) from existing denovo assembly contigs of the 18 non-

7 reference founder genomes to the reference (Col-0 TAIR10) to identify contigs

8 split across the source and sink locus. After alignment we excluded genomic

9 regions with annotated repeats or transposons and alignments that mapped to

10 over 5 genomic loci. We found 2,619 contigs with alignments split into disjoint

11 pieces over 420 QTLs' sources and sinks, suggesting a cut-and-paste

12 mechanism. We also found 460,656 (8.3\%) shared contigs whose alignments

13 overlapped between source and sink regions (duplications, transposons,

14 Microhomology-Mediated Break-Induced Replication (MMBIR) sites and Non-

15 Allelic Homologous Recombination (NAHR) being possible explanations). We

16 randomized the SV-QTLs by circular genome permutation ${ }^{46}$ to determine

17 whether such split and shared contig alignments are overrepresented near

18 SV-QTLs. In particular, for each SV-QTL $i$, if $a(i), b(i)$ are the original position

19 of the source and sink respectively, then a permuted SV-QTL $a_{k}(i), b_{k}(i)$ is 20 defined as:

$$
\begin{gathered}
a_{k}=\left(a(i)+\theta_{k}\right) \bmod L \\
b_{k}=\left(b(i)+\theta_{k}\right) \bmod L \\
\theta_{k} \sim \operatorname{Unif}(0, L)
\end{gathered}
$$


1 with the requirement that $a_{k}(i), b_{k}(i)$ must be on the same chromosome for

2 cis SV-QTLs. We then computed one-sided p-values $\pi_{\text {split }}, \pi_{\text {shared }}$. At the $1 \%$

3 level, trans SV-QTLs were enriched for both split and shared alignments and

4 cis only for split.

5 Validation by PCR We designed PCR primers for 77 breakpoints from 44 SV-

6 QTLs predicted from denovo contigs. We considered two types of

7 experiments: type I experiments had primer oligos corresponding to remote or

8 inverted reference loci so PCR should produce a product in SV genomes and

9 not in the reference; type /I experiment is a control experiment with the

10 reverse outcome (product in the reference, but not in SV genomes). In total,

11 we designed 96 type 1 experiments, one for each of the 77 breakpoints, and

1219 control (type 2) experiments, wherever possible.

13 We designed 20-30bp primer oligos based on the reference (TAIR10), using

14 Primer $3^{47}$, after masking out repeats, transposons and known polymorphisms.

15 SVs tend to be near such sequence features, so we had to relax the default

16 Primer3 criteria to detect oligos, and in particular we required: (i) Maximum

17 allowed product $1.5 \mathrm{~kb}$ (ii) Annealing temperature $10-90^{\circ} \mathrm{C}$ (iii) GC-content 10 -

$1890 \%$ (iv) Self-complementarity 8bp. Primer specificity was tested by BLAT ${ }^{45}$.

19 In 30 (66.6\%) SV-QTLs (46 type 1 experiments) at least one breakpoint was

20 confirmed, i.e. there was at least one type 1 experiment which amplified in a

21 subset of founder genomes (those carrying the SV) while not producing a

22 product in the reference, as expected. In a further 7 SV-QTLs (15.6\%) (15

23 type 1 experiments) the founders carrying an SV-QTL amplified successfully, 
1 but the reference genome also amplified unexpectedly. This may be due to

2 the presence of highly similar sequence nearby, causing unexpected binding

3 of one of the primers - potentially in the presence of duplications. Indeed in 10

4 of these experiments evidence of duplications (multiple bands produced by

5 PCR) was detected in more than two founder genomes. However, in all 15

6 experiments there were at least three founder genomes behaving differently

7 than the reference, indicating that the region is probably structurally variant,

8 although the type of variant may be different to the one predicted by the

9 mapping. We conclude that these results, despite ambiguous, probably

10 indicate SVs, albeit likely polymorphic or of different type than originally

11 predicted. The remaining 16 type 1 experiments failed amplify in any founder.

12 Of the 19 type 2 experiments, 16 succeeded (worked as expected), 2 were

13 ambiguous and 1 failed.

14 In total we confirm at least $30(66.6 \%)$ SV-QTLs with at least one breakpoint, 15 while for a further $7(15.6 \%)$ we have evidence of structural variation - in total 16 up to $82.2 \%$ of the tested SV-QTLs are confirmed.

17 Association with physiological phenotypes. For each of the six read 18 anomaly categories, we computed Pearson correlations and corresponding p19 values between 9 physiological phenotypes and the 11,915 traits measured 20 genome-wide. We selected significant correlations with $\log \mathrm{P}>4$. After filtering 21 out correlations driven by outliers (i.e. in which removal of the three most 22 extreme samples reduced the correlation below the significance threshold) we 23 found 549 traits associated with 40 phenotypes. Each physiological 
1 phenotype had on average 1.56 associated SV traits of the same anomaly

2 type.

3 The effect of SVs on each phenotype was measured by a heritability-like

4 measure, $h_{S V}^{2}$, estimated by linear models. Let $y$ be the vector of phenotypic

5 values for a physiological phenotype with $k$ correlated SV traits (of the same

6 type): $X_{1}, \ldots, X_{k}$, represented by the matrix $X$. The phenotype is modelled as:

$$
y=X a+e
$$

7 The $k$ parameters $a$ were estimated using the $\mathrm{R}$ function $\mathrm{g} \operatorname{lm}()$ and we

8 computed the residual sum of squares RSS and the total sum of squares (i.e.

9 variance of $y$ ) TSS. We also computed the individual effect sizes of all traits

10 contributing to the heritability, by fitting simple linear regression models.

11 Based on this analysis SV traits can explain up to $33 \%$ of the total phenotypic

12 variance.

14 We mapped QTLs for the phenotype residuals after regressing all/each 15 associated SV traits of the same type and compared them to the phenotype 16 QTLs.

18 Published phenotypes We used flowering time and rosette diameter data 19 from a field experiment ${ }^{33}$, as well as phenotypes described in previously ${ }^{25}$.

21 Phenotyping resistance Three replicates of each MAGIC line were grown at

22 the University of Bath in 2.5 inch plastic pots. Plants were monitored daily and 23 germination and bolting day recorded. After plants senesced, the 
1 inflorescence height and the total number of branches were measured. In a

2 separate experiment, MAGIC lines were grown in growth chambers in P24

3 plastic trays and sprayed with $A$. laibachii race $\mathrm{Nc}^{3} 4^{35}$ when plants were 21

4 days old. Nc14 zoospores were suspended in water at a concentration of

$510^{5}$ spores per $\mathrm{ml}$ and incubated on ice for $30 \mathrm{~min}$. The spore suspension was

6 then sprayed on plants using a spray gun, and plants were incubated in a cold

7 room in the dark overnight. Infected plants were kept in a growth chamber

8 under 10 -h light and $14-\mathrm{h}$ dark cycles with a $20^{\circ} \mathrm{C}$ day and $16^{\circ} \mathrm{C}$ night

9 temperature ${ }^{48}$. Resistance was defined as absence of pustules on the leaves

10 at 7 days after inoculation. To minimize errors in scoring, resistant plants were

11 monitored up to 14 days after inoculation. The experiment was reproduced 12 twice.

14 Collection of RNA We obtained a subset of MAGIC lines from the 15 Nottingham Arabidopsis Stock Centre (NASC). We grew 209 of the MAGIC 16 lines at $20^{\circ} \mathrm{C}$ in Percival environmental chambers (Perry, IA, USA) and 17 prepared total RNA as previously described for an earlier study with the 18 MAGIC parental founders ${ }^{8}$; briefly, twenty aerial rosettes from seedlings at the 19 fourth true leaf stage were pooled ${ }^{8}$. RNAseq library construction and 20 sequencing was performed at the Oxford Genomics Centre (Oxford, UK) to 21 produce $2 \times 100 \mathrm{bp}$ reads using the Illumina non-strand specific method. Per 22 Illumina HiSeq lane, samples were barcoded and run in 13-plexes to give 23 approximately 14 million reads per sample. 
1 Alignment of RNAseq reads and expression quantification All libraries

2 were aligned to the TAIR10 reference genome using PALMapper $\mathrm{v} 0.6^{49}$,

3 following a variation-aware alignment approach. Genome variants collected

4 from the 19 founder strains as well as variants reported for a diverse natural

5 population $^{28}$ were integrated and provided to the aligner as set of known

6 variants. Briefly, the mapper used this set of variants in alignments to prevent

7 reference biases in RNAseq read mapping (see previous work ${ }^{8}$ for a

8 rationale). To facilitate accurate alignments, we further provided splice

9 junction information collected in an earlier study with the founder strains ${ }^{8}$ as

10 well as junction information extracted from the TAIR10 genome annotation.

11 The full alignment parameter set for PALMapper was: -M 3 -G 0 -E 3 -I 12 -L

1214 -K 12 -C 14 - I 5000 -NI 1 -SA 5 -UA 50 -CT 50 -JA 15 -J 1 -z 10 -S -seed-

13 hit-truncate-threshold 100 -report-map-read -report-spliced-read -report-map-

14 region -report-splice-sites 0.9 -filter-max-mismatches 0 -filter-max-gaps 0 -

15 filter-splice-region 5 -min-spliced-segment-len 1 -qpalma-use-map-max-len 10

16 -f bam -qpalma-prb-offset-fix -junction-remapping <junction_file> -score-

17 annotated-splice-sites <junction_file> -max-dp-deletions 2 -use-variants-

18 editop-filter -use-variants <variant_file> -filter-variants-minuse 1 -merge-

19 variant-source-ids -use-iupac-snp-variants -filter-variants-map-window 20 -

20 iupac-genome -filter-variants-maxlen 100 -index-precache

22 Gene expression quantification. We used a custom python script that

23 counted the number of reads overlapping with at least one exonic position of

24 an annotated gene feature. For each read only the best alignment was 
1 considered for counting. An alignment was excluded from being counted

2 towards the expression of a gene if (i) at least one position in the alignment

3 overlapped to an annotated intron, (ii) the alignment fell entirely into a region

4 where two or more annotated genes overlap, and (iii) did not start at a position

5 that is part of an exon in all annotated isoforms. For each gene feature the

6 total number of reads passing these filters were used as the expression count.

8 Effects of SVs on gene expression We considered SVs with accurate

9 breakpoints (see Validation by denovo contigs). 119 TAIR10 genes

10 spanned SV breakpoints (i.e. were disrupted by SVs) and 6,909 were inside

11 them (transposed, inverted or duplicated). Genes were divided into three

12 categories: disrupted by breakpoints, within SV-regions and outside SVs and

13 compared with respect to mean and variance using t-tests. We also computed

14 the correlation of these genes with their local read anomaly values (for the six

15 read anomaly types), i.e. with the $10 \mathrm{~kb}$ source region that contains the gene

16 and compared the mean and variance (by a t-test and an F-test, respectively)

17 of the Pearson correlation coefficients across categories.

18 Acknowledgements

$20 \mathrm{MI}, \mathrm{RM}$ were supported by the Wellcome Trust Core Award Grant Number

21 090532/Z/09/Z. RMC was supported by NSF 0929262. EJO and RG were

22 supported by National Institutes of Health Genetics Training Grant T32

23 GM07464. We thank Fernando Rabanal for comments on the manuscript. 


\section{References}

1. Simpson, J. T. \& Pop, M. The Theory and Practice of Genome Sequence Assembly. Annu. Rev. Genomics Hum. Genet. (2015). doi:10.1146/annurev-genom-090314-050032

2. Chaisson, M. J. \& Tesler, G. Mapping single molecule sequencing reads using basic local alignment with successive refinement (BLASR): application and theory. BMC Bioinformatics 13, 238 (2012).

3. Jain, M. et al. Improved data analysis for the MinION nanopore sequencer. Nat. Methods (2015). doi:10.1038/nmeth.3290

4. Cao, J. et al. Whole-genome sequencing of multiple Arabidopsis thaliana populations. Nat Genet 43, 956-963

5. Mills, R. E. et al. Mapping copy number variation by population-scale genome sequencing. Nature 470, 59-65 (2011).

6. McKenna, A. et al. The Genome Analysis Toolkit: a MapReduce framework for analyzing next-generation DNA sequencing data. Genome Res. 20, 1297-1303 (2010).

7. Rimmer, A. et al. Integrating mapping-, assembly- and haplotype-based approaches for calling variants in clinical sequencing applications. Nat. Genet. 46, 912-918 (2014).

8. Gan, X. et al. Multiple reference genomes and transcriptomes for Arabidopsis thaliana. Nature 477, 419-423 (2011).

9. Ye, K., Schulz, M. H., Long, Q., Apweiler, R. \& Ning, Z. Pindel: A pattern growth approach to detect break points of large deletions and medium 
sized insertions from paired-end short reads. Bioinformatics 25, 28652871 (2009).

10. Chen, K. et al. BreakDancer: an algorithm for high-resolution mapping of genomic structural variation. Nat. Methods 6, 677-681 (2009).

11. Simpson, J. T., Mclntyre, R. E., Adams, D. J. \& Durbin, R. Copy number variant detection in inbred strains from short read sequence data.

12. Manske, H. M. \& Kwiatkowski, D. P. LookSeq: A browser-based viewer for deep sequencing data. Genome Res. 19, 2125-2132 (2009).

13. Sindi, S. S., Onal, S., Peng, L. C., Wu, H.-T. \& Raphael, B. J. An integrative probabilistic model for identification of structural variation in

14. Rausch, T. et al. DELLY: structural variant discovery by integrated paired-end and split-read analysis. Bioinformatics 28, i333-i339 (2012).

15. Layer, R. M., Chiang, C., Quinlan, A. R. \& Hall, I. M. LUMPY: a

16. Kronenberg, Z. N. et al. Wham: Identifying Structural Variants of Biological Consequence. PLoS Comput. Biol. 11, e1004572 (2015).

17. Durbin, R. M. et al. A map of human genome variation from populationscale sequencing. Nature 467, 1061-1073 (2010).

18. Cai, N. et al. Sparse whole-genome sequencing identifies two loci for major depressive disorder. Nature (2015). doi:10.1038/nature14659 
1 19. Nicod, J. et al. Genome-wide association of multiple complex traits in outbred mice by ultra-low-coverage sequencing. Nat. Genet. (2016). doi:10.1038/ng.3595

20. Davies, R. W., Flint, J., Myers, S. \& Mott, R. Rapid genotype imputation from sequence without reference panels. Nat. Genet. 48, 965-9 (2016).

21. Yalcin, B. et al. Sequence-based characterization of structural variation in the mouse genome. Nature 477, 326-329 (2011).

22. Cao, J. et al. A whole-genome map of sequence variants in multiple Arabidopsis thaliana populations. Nat Genet In Press, (2011).

23. Hu, T. T. et al. The Arabidopsis lyrata genome sequence and the basis of rapid genome size change. Nat. Genet. 43, 476-81 (2011).

24. Quadrana, L. et al. The Arabidopsis thaliana mobilome and its impact at the species level. Elife 5, e15716 (2016).

25. Kover, P. X. et al. A Multiparent Advanced Generation Inter-Cross to fine-map quantitative traits in Arabidopsis thaliana. Plos Genet. 5, e1000551 (2009)

26. Li, H. \& Durbin, R. Fast and accurate long-read alignment with BurrowsWheeler transform. Bioinformatics 26, 589-595 (2010).

27. Lunter, G. \& Goodson, M. Stampy: a statistical algorithm for sensitive and fast mapping of Illumina sequence reads. Genome Res 21, 936939 (2011).

28. Long, Q. et al. Massive genomic variation and strong selection in Arabidopsis thaliana lines from Sweden. Nat. Genet. 45, 884-90 (2013). 
1 29. Fransz, P. F. et al. Integrated cytogenetic map of chromosome arm 4S of A. thaliana: structural organization of heterochromatic knob and centromere region. Cell 100, 367-376 (2000).

30. Wijnker, E. et al. The genomic landscape of meiotic crossovers and gene conversions in Arabidopsis thaliana. Elife 2, e01426 (2013).

31. Lai, A. G., Denton-Giles, M., Mueller-Roeber, B., Schippers, J. H. \& Dijkwel, P. P. Positional information resolves structural variations and uncovers an evolutionarily divergent genetic locus in accessions of Arabidopsis thaliana. Genome Biol Evol (2011). doi:evr038 [pii]10.1093/gbe/evr038

32. Berlin, K. et al. Assembling large genomes with single-molecule sequencing and locality-sensitive hashing. Nat. Biotechnol. 33, 623-630 (2015)

33. Springate, D. A. \& Kover, P. X. Plant responses to elevated temperatures: a field study on phenological sensitivity and fitness responses to simulated climate warming. Glob. Chang. Biol. 20, 456-65 (2014).

34. Nishimura, N. et al. ABA hypersensitive germination2-1 causes the activation of both abscisic acid and salicylic acid responses in Arabidopsis. Plant Cell Physiol. 50, 2112-22 (2009).

35. Thines, M. et al. A new species of Albugo parasitic to Arabidopsis thaliana reveals new evolutionary patterns in white blister rusts (Albuginaceae). Persoonia Mol. Phylogeny Evol. Fungi 22, 123-128 
(2009).

36. Van Der Biezen, E. A., Freddie, C. T., Kahn, K., Parker, J. E. \& Jones, J. D. G. Arabidopsis RPP4 is a member of the RPP5 multigene family of TIR-NB-LRR genes and confers downy mildew resistance through multiple signalling components. Plant J. 29, 439-451 (2002).

37. Yi, H. \& Richards, E. J. Gene duplication and hypermutation of the pathogen Resistance gene SNC1 in the arabidopsis bal variant. Genetics 183, 1227-1234 (2009).

38. Kang, H. M. et al. Efficient control of population structure in model organism association mapping. Genetics 178, 1709-1723 (2008).

39. Kang, H. M., Ye, C. \& Eskin, E. Accurate discovery of expression quantitative trait loci under confounding from spurious and genuine regulatory hotspots. Genetics 180, 1909-1925 (2008).

40. Durkin, K. et al. Serial translocation by means of circular intermediates underlies colour sidedness in cattle. Nature 482, 81-84 (2012).

41. Genovese, G. et al. Using population admixture to help complete maps of the human genome. Nat. Genet. 45, 406-14, 414-2 (2013).

42. Li, J.-Y., Wang, J. \& Zeigler, R. S. The 3,000 rice genomes project: new opportunities and challenges for future rice research. Gigascience 3, 8 (2014)

43. Alonso-Blanco, C. et al. 1,135 Genomes Reveal the Global Pattern of Polymorphism in Arabidopsis thaliana. Cell (2016). doi:10.1016/j.cell.2016.05.063 
1 44. Ito, H. \& Kakutani, T. Control of transposable elements in Arabidopsis thaliana. Chromosom. Res. 22, 217-223 (2014).

3 45. Kent, W. J. BLAT--the BLAST-like alignment tool. Genome Res. 12, 656-664 (2002).

46. Cabrera, C. P. et al. Uncovering networks from genome-wide association studies via circular genomic permutation. G3 (Bethesda). 2, 1067-75 (2012).

47. Rozen, S. \& Skaletsky, H. Primer3 on the WWW for general users and for biologist programmers. Methods Mol. Biol. 132, 365-386 (2000).

48. Kemen, E. et al. Gene gain and loss during evolution of obligate parasitism in the white rust pathogen of Arabidopsis thaliana. PLoS Biol. 9, (2011).

49. Jean, G., Kahles, A., Sreedharan, V. T., De Bona, F. \& Ratsch, G. RNASeq read alignments with PALMapper. Curr Protoc Bioinforma. Chapter 11, Unit 116 


\section{Supplementary Figures legends}

2 Figure S1 Manhattan plots and founder contributions for high read coverage

3 in a cis $(\mathbf{a}, \mathbf{c})$ and a trans $(\mathbf{b}, \mathbf{d})$ SV-QTL. In the manhattan plots the red line

4 shows the source and the association peak the sink of the SV-QTL. In the

5 founder contributions tables rows and columns correspond to founder

6 haplotypes at the sink and source, respectively. The colour hue at each cell is

7 the trait value for each combination of founder haplotypes, darker colour

8 means higher value. In c trait values range from 0 to 1000. The figure shows a

9 duplication (confirmed by denovo contigs) present in 3 founders, namely Bur-

100 , Col-0 and Edi-0. In d trait values (high read coverage) range from 0 to 600

11 and the figure is showing a trans QTL in chromosome 5, present in Bur-0, Oy-

12 0, Po-0, Rsch-4 and Wu-0.

13 Figure S2 Distribution of SV allele frequencies, defined as the fraction of 14 founders carrying the SV allele at SV-QTLs.

15 Figure S3 Alignment of a $175 \mathrm{~kb}$ manually assembled contig from Ler-0, 16 chr5:22.05 - 25.23Mb $\mathrm{Mb}^{31}$ to the reference. See legend of Figure 3 for 17 explanation.

Figure S4 Validation of SV QTL predictions using paired-end data from high

19 coverage sequence in the 19 founder genomes. The figure shows results for 2,391 SV-QTLs for which predictions of founder haplotypes carrying SVs were

21 reliable. Each bar corresponds to a subsample of the SV QTLs with maximum 22 genome-wide logP exceeding a given threshold, with bar height showing the 
1 fraction that were supported by reads at $P<0.05$. The right-most bar shows the

2 results in the entire set of 2,391 QTLs. The test compares the number of

3 reads linking the $10 \mathrm{~kb}$ source region to a variably sized window around the

4 sink (see Methods). The colours are coding the different window lengths used

5 - where different window sizes gave significant results for the same SV QTL

6 we report the smallest. 


\section{Supplementary Table legends}

2 Table S1 The 19 founder accessions of the MAGIC population of

3 Recombinant Inbred Lines. Shown are the stock centre numbers, the

4 accessions' names and the place of origin.

5 Table S2 The mosaic reconstructions of 488 MAGIC lines. Each row

6 represents one segment of a MAGIC line. magic: name of the MAGIC line;

7 chr: the chromosome of the segment; acc: the founder accession present at

8 this locus; from.bp, to.bp: the start and end bp coordinates of the segment

9 (TAIR10); from.site, to.site: the start and end position in terms of sites;

10 len.bp: the length of the segment in bp; sites: the length of the segment in

11 sites; errors: the number of errors (sites whose genotype does not agree with

12 the founder accession genotype); error.site: the number of errors divided by

13 the number of sites; error.bp: the number of errors divided by the length of

14 the segment

Table S3: Catalogue of SV-QTLs detected by genetic mapping of read pair

17 anomalies. Each row represents a distinct SV. SV-QTLs with coincident

18 sources and sinks for different read pair anomalies are merged into a single

19 row. src.chr, src.pos: genomic location of the source, defined as the start of

20 the 10kb region in which read pair anomalies were measured; sink.chr,

21 sink.pos: genomic location of the sink, defined as the peak of association;

22 read.anomaly.traits: read anomaly traits with the sink SV-QTL;

23 max.gw.logp: maximum genome-wide $\log \mathrm{P}$ estimated by the genome scan 
1 ( $\lambda_{A}$, see Methods), fitted.p - extreme value distribution fitted $p$-value $\left(\gamma_{A}\right.$ - see

2 Methods), - qtl.distance: cis or trans; SV.type: prediction of the type of

3 structural variant based on the read anomaly traits that had a SV-QTL ;

4 sink.founders - founder haplotypes predicted to carry the SV, NA means

5 unknown (Methods); known.indel.dist: distance between the midpoints of

6 the source loci to the nearest large (>100bp) SV from ${ }^{17}$;

7 read.support.founder: P-value of FET comparing read pairs connecting the

8 source and the sink in founders carrying the SV allele from the high-coverage

9 sequencing of the 19 founders (NA if founders are the sink were unknown) ${ }^{17}$,

10 read.support.founder.window: size of window (distance from association

11 peak) containing the significant association in read.support.founder,

12 read.support.line, read.support.line.window: same validation test using

13 read pairs from the 488 MAGIC lines; denovo.contigs: Boolean variable

14 showing confirmation by at least one denovo contig.

16 Table S4. Breakpoints of 420 SVs detected using denovo contigs. founder:

17 founder genome in which the breakpoint was detected; source.chr, 18 source.pos: source position in which the read anomalies were measured;

19 sink.chr, sink.pos: SV-QTL position; source.break.from, source.break.to:

20 breakpoints detected by denovo contigs corresponding to the source area;

21 sink.break.from, sink.break.to: breakpoints corresponding to sink;

22 source.length, sink.length: length of the structurally variant region in the

23 source and sink regions. 
1 Table S5. PCR validation results (a) Results per SV-QTL. QTL_ID: id of the

2 SV-QTL; QTL: coordinates of the tested SV-QTL, position of the source

3 followed by the position of the sink; confirm: $\mathrm{Y}-$ yes, $\mathrm{A}-$ ambiguous, $\mathrm{N}-\mathrm{no}$;

4 dist: Boolean indicator of whether the SV-QTL is cis or trans; read pred: type

5 of the SV (e.g. transposition, indel etc) predicted by read anomalies, PCR

6 pred: prediction of the type of SV based on the contigs and PCR results. (b)

7 Results per experiment. Each row corresponds to a single experiment (unique

8 combination of primers) performed on all 19 founders. QTL_ID: ID of the SV-

9 QTL predicted; type: type of experiment 1 or 2 (see Methods); Forward,

10 Reverse: unique identifier of the primers used, in the form

11 chr_pos_orientation, (e.g. id 4_1853989F means the sequence starting from

12 chr 4, 1853989bp and with forward orientation). The identifier INV means that

13 the sequence has the opposite orientation than expected. The remaining 14 columns correspond to each of the founder genomes, 1 meaning that the 15 experiment amplified, 0 that it did not, and $>1$ that it produced multiple bands.

16 (c) Primer sequences.

Table S6 Nine physiological traits measured in MAGIC lines that were used in 19 this study.

Table S7. Effects of SVs on physiological phenotypes. Phenotype:

21 physiological phenotype; SV trait type: type of read pair anomaly, SV-QTL:

22 number of SV traits that have SV-QTLs, trans: number of trans SV-QTLs;

23 max.source: position of the source of the maximum-contributing SV trait;

24 max.sink: position of the sink QTL of the maximum-contributing SV, or NA if it 
1 is not mapped, physio.QTL: position of the QTL of the physiological

2 phenotype; overlap.source, overlap.sink: Boolean indicator of whether the

3 source or sink of the SV trait overlaps (within $200 \mathrm{~kb}$ ) with the QTL of the

4 physiological phenotype, NA if there is no QTL.

5 Table S8. Association of SVs with gene expression. gene - gene id, chrom,

6 start, end - gene coordinates, Same QTL: position of the gene relative to

7 SVs (break: the gene is spanning an SV breakpoint, within: the gene is

8 between SV breakpoints so it may be transposed, inverted or duplicated,

9 outside: the gene lies outside SVs), mean: mean gene expression, var:

10 variance of gene expression, zeroes: proportion of silenced (zero expression)

11 transcripts, r_unmapped_largeisize, r_impop_paired_LT, r_same_strand,

12 r_largeisize, r_excess_reads, r_unmapped: Pearson correlation

13 coefficients between expression levels and values of the local read anomaly

14 trait, for each of the six anomaly types. 